\title{
S29434, a Quinone Reductase 2 Inhibitor: Main Biochemical and Cellular Characterization ${ }^{\text {[ }}$
}

\author{
Jean A. Boutin, ${ }^{1}$ Frederic Bouillaud, Elzbieta Janda, István Gacsalyi, ${ }^{2}$ Gérald Guillaumet \\ Etienne C. Hirsch, Daniel A. Kane, Françoise Nepveu, Karine Reybier, Philippe Dupuis, \\ Marc Bertrand, Monivan Chhour, Thierry Le Diguarher, Mathias Antoine, Karen Brebner, \\ Hervé Da Costa, Pierre Ducrot, Adeline Giganti, Vishalgiri Goswami, Hala Guedouari, \\ Patrick P. Michel, Aakash Patel, Jérôme Paysant, Johann Stojko, Marie-Claude Viaud- \\ Massuard, and Gilles Ferry
}

Pôle d'Expertise Biotechnologie, Chimie \& Biologie, Institut de Recherches SERVIER, Croissy-sur-Seine, France (J.A.B., M.A., Pi.D., A.G., J.S., G.F.); Institut Cochin, INSERM U1016, CNRS-UMR8104, Université Paris Descartes, Paris, France (F.B., H.G.); Department of Health Sciences, Magna Graecia University, Catanzaro, Italy (E.J.); Egis Pharmaceuticals PLC, Budapest, Hungary (I.G.); Institut de Chimie Organique et Analytique (ICOA), Université d'Orléans, UMR CNRS 7311, Orléans Cedex 2, France (G.G., H.D.C.); Institut du Cerveau et de la Moelle épinière, ICM, Inserm U 1127, CNRS UMR 7225, Sorbonne Université, Paris, France (E.C.H., P.P.M.); Departments of Human Kinetics (D.A.K.) and Psychology (K.B.), St. Francis Xavier University, Antigonish, Nova Scotia, Canada; UMR 152 Pharma-Dev, Université de Toulouse, IRD, UPS, Toulouse, France (F.N., K.R., M.C.); EUROFINS-CEREP SA, Celle L'Evescault, France (Ph.D.); Technologie Servier, Orléans, France (M.B., T.L.D.); CNRS-UMR 7292, GICC Innovation Moléculaire et Thérapeutique, Université de Tours, Tours, France (H.D.C., M.-C.V.-M.); Oxygen Healthcare Pvt Ltd, Ahmedabad, Gujarat, India (V.G., A.P.); and Pôle d'Innovation Thérapeutique de Cardiologie, Institut de Recherches SERVIER, Suresnes, France (J.P.)

Received September 3, 2018; accepted December 17, 2018

\section{ABSTRACT}

Quinone reductase 2 (QR2, E.C. 1.10.5.1) is an enzyme with a feature that has attracted attention for several decades: in standard conditions, instead of recognizing $\mathrm{NAD}(\mathrm{P}) \mathrm{H}$ as an electron donor, it recognizes putative metabolites of $\mathrm{NADH}$, such as $N$-methyl- and $N$-ribosyl-dihydronicotinamide. QR2 has been particularly associated with reactive oxygen species and memory, strongly suggesting a link among QR2 (as a possible key element in pro-oxidation), autophagy, and neurodegeneration. In molecular and cellular pharmacology, understanding physiopathological associations can be difficult because of a lack of specific and powerful tools. Here, we present a thorough description of the potent, nanomolar inhibitor
[2-(2-methoxy-5H-1,4b,9-triaza(indeno[2,1-a]inden-10-yl)ethyl]2-furamide (S29434 or NMDPEF; IC $5_{50}=5-16 \mathrm{nM}$ ) of QR2 at different organizational levels. We provide full detailed syntheses, describe its cocrystallization with and behavior at QR2 on a millisecond timeline, show that it penetrates cell membranes and inhibits QR2-mediated reactive oxygen species (ROS) production within the $100 \mathrm{nM}$ range, and describe its actions in several in vivo models and lack of actions in various ROS-producing systems. The inhibitor is fairly stable in vivo, penetrates cells, specifically inhibits QR2, and shows activities that suggest a key role for this enzyme in different pathologic conditions, including neurodegenerative diseases.

\section{Introduction}

Quinone reductases, described as early as 1954 in peas, are pyridine nucleotide-dependent enzymes involved in the

E.C.H. and P.P.M. acknowledge the support of the funding programs "Investissements d'avenir" ANR-10-IAIHU-06 and "Investissements d'avenir" ANR-11-INBS-0011-NeurATRIS: Translational Research Infrastructure for Biotherapies in Neurosciences. This work benefited from equipment and services from the CELIS core facility (Institut du Cerveau et de la Moelle Epinière, Paris). Dr. D.A.K. acknowledges support from the Canada Foundation for Innovation and the Natural Sciences and Engineering Research Council of Canada. Dr. E.J. received financial support from Herbal and Antioxidant Derivatives srl, Biano (RC), Italy, and from FFARB 2017 (Basic Research Activities Fund).

${ }^{1}$ Current affiliation: Institut de Recherches Internationales Servier, Suresnes, France.

${ }^{2}$ Current affiliation: ATRC Aurigon Ltd., Budapest, Hungary.

https://doi.org/10.1124/mol.118.114231.

S This article has supplemental material available at molpharm. aspetjournals.org. detoxification of natural quinones (Wosilait and Nason, 1954). Conover and Ernster (1960) first described the reference quinone reductase enzyme, initially designated as DT-diaphorase, in mammals. In the 1990s, another quinone reductase was cloned: quinone reductase 2 (QR2 or NQO2), which has an unusual history (Zhao et al., 1997). The initial description indicated that QR2 was a strict analog of quinone reductase 1 [DT-diaphorase, or $\mathrm{NAD}(\mathrm{P}) \mathrm{H}$ dehydrogenase (quinone 1) (QR1), NQO1, E.C. 1.6.5.2] with similar properties. Later findings revealed, however, that it was quite different. Research in the 1960s identified QR2 as the only reductase using noncanonical cosubstrates such as $N$-ribosyland $N$-methyl-dihydronicotinamide and para-quinones as substrates (Liao and Williams-Ashman, 1961; Liao et al., 1962) but not NADH or NADPH (Zhao et al., 1997; Ferry et al., 2010). Later, Zhao et al. (1997) cloned and crystallized QR2, but its physiologic role and specificity were poorly documented 
and understood, except that it recognized nonquinone compounds, such as CB1954 (5-(1-aziridinyl)-2,4-dinitrobenzamide), as a substrate (Wu et al., 1997). This finding was the basis of efforts to develop CB1954 as a prodrug with interesting cytotoxic properties (Knox et al., 2003). QR2 has been implicated in effects distantly related to quinone metabolism, such as antimalarial action (Kwiek et al., 2004; Cassagnes et al., 2017 ), the AMPA ( $\alpha$-Amino-3-hydroxy-5-methylisoxazole-4propionic acid hydrate) signaling pathway (Rappaport et al., 2015), paraquat-mediated toxicity (Janda et al., 2013, 2015), oxidative stress sensing (Leung and Shilton, 2013), and oocyte maturation (Chen et al., 2017).

In the search for the melatonin MT3 binding site, described by Duncan et al. (1988) and our team (Paul et al., 1999), we found that the binding site was, in fact, in QR2 [Nosjean et al., 2000; also see discussion by Boutin and Ferry (2018)]. A screen of about 20,000 compounds from our library, comprising natural products such as flavonoids (Boutin et al., 2005), added further candidates to the list of QR2 inhibitors. Already on this list were resveratrol (Buryanovskyy et al., 2004), tacrine (den Braver-Sewradj et al., 2018), chloroquine (CLQ) (Kwiek et al., 2004), dabigatran (a prescription thrombin inhibitor) (Michaelis et al., 2012), and tetracyclic compounds (Boussard et al., 2006). Some products of quinone reduction are fairly unstable, especially in the absence of a conjugating system(s), so the reaction products (quinols) may spontaneously yield back the original quinones in the presence of oxygen. Similar quinone cycling has been described for QR1 (Bindoli et al., 1990; Nutter et al., 1992; Bian et al., 2017). Without the cellular conjugating capacities that neutralize the process by conjugating the quinol (e.g., menadiol) with glucuronic acid (Kappus and Sies, 1981; Bolton et al., 2000; Nishiyama et al., 2010), the by-product of this futile cycle is the production of reactive oxygen species (ROS). Because electron paramagnetic resonance (EPR) spectroscopy can detect ROS generation (Reybier et al., 2011), we used this method to show that, under some conditions, the pro-oxidant property of QR2 can be confirmed and measured.

Many organs and cells express QR2 (Nosjean et al., 2001), and it might have a key role in several pathologic conditions (Boutin, 2016). Given this potential, we assessed the specificity of the QR2 inhibitor [2-(2-methoxy-5H-1,4b,9-triaza(indeno[2,1a]inden-10-yl)ethyl]-2-furamide (S29434) and its actions in different contexts, adding to the already available data to consolidate its use as an established QR2 inhibitor tool. The present paper gives the full description of the characteristics of S29434. Despite several publications on this inhibitor, no report has described its synthesis, specificity for alternative enzymes or receptors, or characteristics of its stability in living systems. Thus, we generated as much information as possible about S29434 to make its use more appropriate for QR2 pharmacology. Here, we cover its most important characteristics, from its chemistry and molecular and cellular pharmacology to its specificity for other ROSgenerating systems and its in vivo activities. These data illustrate that S29434 or unknown metabolites reach QR2 and inhibit this enzyme in situ. Furthermore, its specificity seems to be limited to QR2, making this compound a valuable tool for understanding the role of QR2 in multiple scenarios, even in vivo.

\section{Materials and Methods}

\section{Chemical Syntheses}

S29434 synthesis has never been published. In this study, two different paths were explored for its synthesis. Both are summarized in Figs. 1 and 2. The synthetic routes are given in full detail in the Supplemental Materials, and the various steps to obtain the compounds are detailed in the Supplemental Figure S1. The final analyses of the product obtained with the second route are available as Supplemental Data 1 (spectral analyses of compounds 3, 4, and S29434 obtained by the first synthetic route). All data obtained (analytical, spectral, biophysical) on the compound were consistent with its structure. S29434 was obtained with purity above 99\% as adjudged by liquid chromatography-mass spectrometry and NMR (see Supplemental Analytical Data). Analytical details of the second route (Supplemental Fig. S2) are also given as Supplemental Data 2-7.

\section{QR2 Inhibition Measurements}

The potency of S29434 was measured in standard kinetic assays. In brief, QR2 enzymatic activity was measured under FAD saturation using substrate (menadione) and $100 \mu \mathrm{M}$ cosubstrate $N$ benzyldihydronicotinamide (BNAH) or $N$-ribosyldihydronicotinamide $(\mathrm{NRH})$. An oxidoreduction reaction was performed at $25^{\circ} \mathrm{C}$ in a buffer of $50 \mathrm{mM}$ Tris-HCl (pH 8.5), $500 \mathrm{nM}$ FAD, 1 mM octylglucopyranoside, and 5\% dimethylsulfoxide (DMSO). Enzymatic kinetics measured the decrease in cosubstrate absorbance corresponding to its oxidation. This reaction was followed at either $350 \mathrm{~nm}$ (BNAH) or $340 \mathrm{~nm}(\mathrm{NRH})$ on a FLUOstar 384-well plate reader (BMG, Offenburg, Germany). The slope of absorbance decrease was determined using FLUOstar Optima software (BMG) and expressed in units of optic density.s -1 then in M.s-1 using the Beer-Lambert law. This measurement was next corrected from its corresponding nonenzymatic (spontaneous) oxidation rate measured in the absence of enzyme, which corresponds to the specific maximum-activity oxidoreduction reaction. For the inhibitory hQR2 enzymatic activity assay, S29434 was used in the range of $50 \mathrm{pM}$ to $5 \mu \mathrm{M}$. The inhibitory concentration $50 \%\left(\mathrm{IC}_{50}\right)$ and the inhibition percentage of cosubstrate oxidation were determined using the PRISM program (GraphPad Software Inc., San Diego, CA). These experiments were conducted over 50 times (inhibition, Fig. 3A) or in triplicate (Fig. 3B).

\section{Binding Kinetics of S29434 and Resveratrol to QR2 Oxidized FAD}

Transient kinetics were measured at $25^{\circ} \mathrm{C}(\mathrm{S} 29434)$ or $4^{\circ} \mathrm{C}$ (resveratrol) with an SFM-300 stopped-flow mixer fitted with an FC-15 cell and coupled to an MOS-200 M rapid spectrophotometer equipped with a $150 \mathrm{~W} \mathrm{Xe}-\mathrm{Hg}$ lamp (Bio-Logic Science Instruments, Seyssinet-Pariset,

ABBREVIATIONS: AMPA, $\alpha$-Amino-3-hydroxy-5-methylisoxazole-4-propionic acid hydrate; ANOVA, analysis of variance; BBB, blood-brain barrier; BCNU, 1,3-bis(2-chloroethyl)-1-nitrosourea; BNAH, N-benzyldihydronicotinamide; CLQ, chloroquine; DMEM, Dulbecco's modified essential medium; DMPO, 5,5'-dimethyl-1-pyrroline $\mathrm{N}$-oxide; DMSO, dimethylsulfoxide; EPR, electron paramagnetic resonance; ET, exploration time; KO, knockout; MK-801, [5R,10S]-[1]-5-methyl-10,11-dihydro-5H-dibenzo[a,d]cyclohepten-5,10-imine; MPP ${ }^{+}$, 1-methyl-4-phenylpyridinium; NRH, $N$ ribosyldihydronicotinamide; PBS, phosphate-buffered saline; Pen-Strep, penicillin and streptomycin; pGP, P-glycoprotein 1 efflux transporter; QR1, $\mathrm{NAD}(\mathrm{P}) \mathrm{H}$ dehydrogenase (quinone 1), E.C. 1.6.5.2; QR2, quinone reductase 2 or ribosyldihydronicotinamide dehydrogenase (quinone), formerly E.C. 1.10.99.2, now E.C. 1.10.5.1; ROS, reactive oxygen species; S29434, [2-(2-methoxy-5H-1,4b,9-triaza(indeno[2,1-a]inden-10-yl)ethyl]-2-furamide; siRNA, small interfering RNA. 
<smiles>CCOc1ccc(C(=O)NCCc2c[nH]c3ccc(OC)nc23)o1</smiles>

$65 \%$

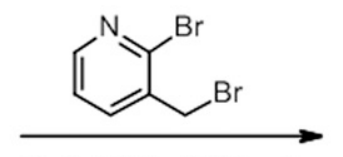

$\mathrm{NaH}, \mathrm{DMF}, 0^{\circ} \mathrm{C}$ to r.t $64 \%$<smiles>COc1ccc2c(n1)c(CCNC(=O)c1ccco1)cn2Cc1cccnc1Br</smiles><smiles>COc1ccc2c(n1)c(CCNC(=O)c1ccco1)c1n2Cc2cccnc2-1</smiles>

5 (S 29434)

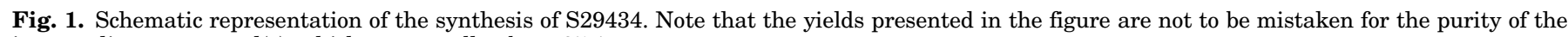
intermediary compound(s), which was usually above $95 \%$.

France). Typical mixing dead time was $2.6 \mathrm{~ms}$. QR2 tryptophans were excited at $295 \mathrm{~nm}$ to minimize photobleaching and excitation of tyrosines. Fluorescence emission was recorded using a 320 -nm cutoff filter combined with a UG11 bandpass filter to block S29434 fluorescence. Binding experiments were performed under pseudo-first-order conditions by mixing an equal volume (75 $\mu \mathrm{l}$ ) of QR2 and ligand in $50 \mathrm{mM}$ Tris-HCl, $1 \mathrm{mM} \beta$-octyl-D-glucopyranoside, and 5\% DMSO (pH 8.5). The QR2 monomer concentration after mixing was $1 \mu \mathrm{M}$. Fluorescence traces were first analyzed individually with the BioKine software (version 4.80; BioLogic) as the sum of up to two exponential terms, as described by eq. 1 , with $n$ being the number of exponentials, $a_{i}$ the amplitude of the ith exponential, $k_{\text {obs }} \mathrm{i}$ the rate constant of the ith exponential, $\mathrm{c}$ the trace endpoint, and bt accounting for the slow linear drift caused by photobleaching. Fig. 5 was generated with Prism (version 7.03; GraphPad):

$$
y=\left(\sum_{\mathrm{i}=1}^{\mathrm{n}} \mathrm{a}_{\mathrm{i}} e^{\left(-k_{\mathrm{obs}} \mathrm{t}\right)}\right)+\mathrm{bt}+\mathrm{c} .
$$

The $k_{\text {obs }}$ values were plotted against the ligand concentration. When a linear increase of $k_{\text {obs }}$ was observed, data were fitted to eq. 2 , describing a one-step binding mechanism, using Prism (version 7.03; GraphPad):

$$
k_{\mathrm{obs}}=k_{1}[\text { ligand }]+k_{-1} .
$$

\section{Specificity Tests}

General Specificity. Most of the tests were subcontracted to Eurofins. All experimental details can be found online on their site (www.eurofinsdiscoveryservices.com).

Kinases. The panel of kinases for screening is available from Eurofins (ExpressDiversityKinase). It comprises 46 different kinases chosen to cover a maximal structural diversity among this large family of over 500 different enzymes. S29434 was tested at $100 \mathrm{nM}$ on the enzymatic activity of all the kinases using a standard radiometric assay.

Sirtuin Experiments. The use of this original technology has been reported in a technical note by Agilent (see https://www.agilent. com/cs/library/applications/5990-9345en_lo.pdf). In brief, recombinant sirtuin 1 was produced in Escherichia coli and purified. The nonacetylated and acetylated Foxo-3 substrates [hFoxo-3a-290-K(Ac): Ac-DSPSQLS-K(Ac)-WPGSPTS-NH2] were synthesized by GENEPEP SA (Saint-Jean-de-Védas, France). Deacetylase reactions were carried out in reaction buffer $(50 \mathrm{mM}$ Tris- $\mathrm{HCl}, 137 \mathrm{mM} \mathrm{NaCl}, 2.7 \mathrm{mM} \mathrm{KCl}$, $1 \mathrm{mM} \mathrm{MgCl} 2,0.05 \%$ bovine serum albumin, $1 \mathrm{mM}$ TCEp, $0.8 \mathrm{mM}$ $\mathrm{NAD}+$ ) and $20 \mathrm{nM}$ sirtuin 1 for 30 minutes at room temperature. The reaction was initiated by the addition of peptide substrate and quenched by the addition of $2 \%$ formic acid. The transition from the peptide substrate (excitation at $829.1 \mathrm{~nm}$; emission at $544.6 \mathrm{~nm}$ ) to the peptide product (excitation at $808.4 \mathrm{~nm}$; emission at $544.5 \mathrm{~nm}$ ) was measured.

NAD(P)H Oxidase Experiments. This enzyme activity was measured using the lucigenin assay as described by Sasaki et al. (2013) and Kuribayashi et al. (2008). This assay was adapted to the U937 cell line as a biologic source.

\section{ROS Production in Acellular and Cellular Experiments Using EPR}

5,5'-Dimethyl-1-pyrroline $N$-oxide (DMPO) was purchased from Interchim (Montluçon, France). Menadione, adrenochrome, 3,3'methylene-bis(4-hydroxycoumarin) (dicoumarol), NADH, and phosphate-buffered saline (PBS) were purchased from SigmaAldrich-Fluka Co. (Saint Quentin Fallavier, France). BNAH was purchased from TCI Europe (Zwijndrecht, Belgium). NRH was custom synthesized by $\mathrm{O} 2 \mathrm{~h}$ (Ahmedabad, India). DMSO was purchased from Fisher (Loughborough, UK). The DMPO stock solution (1 M) was prepared in water and stored at $-80^{\circ} \mathrm{C}$ until required. The inhibitors (S29434 and dicoumarol) were prepared as $1 \mathrm{mM}$ solutions in DMSO and diluted in PBS to yield $0.2 \mathrm{mM}$. The substrate and cosubstrate stock solutions were prepared in DMSO. Experiments on pure enzyme (QR1 or QR2) were performed with demineralized (18 M 2 ) and deaerated water containing $50 \mathrm{mM}$ tris(hydroxymethyl)aminomethane and $1 \mathrm{mM} \beta$-octyl-glucoside, final $\mathrm{pH}$ 8.5. The EPR spectra were recorded immediately after mixing the pure enzyme with the inhibitor when needed (dicoumarol or S29434), DMPO (final concentration $125 \mathrm{mM}$ ), the cosubstrate (NADH or NADPH for QR1 and NRH or $\mathrm{BNAH}$ for $\mathrm{QR} 2$, final concentration $3 \mathrm{mM}$ ), and finally the quinone 


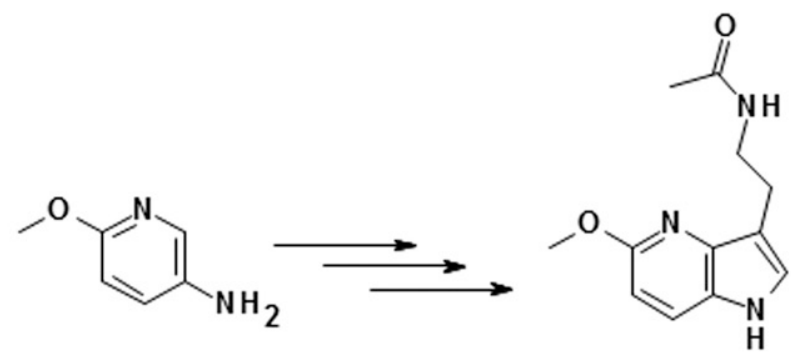

1 $\underline{6}$<smiles>COc1ccc2c(n1)C(CCNC(C)=O)=C1c3ncccc3N1C2</smiles>

$\underline{8}$<smiles>COc1ccc2c(n1)c(CCNC(C)=O)cn2Cc1cccnc1Br</smiles>

1) $10 \% \mathrm{H}_{2} \mathrm{SO}_{4} 100^{\circ} \mathrm{C}$<smiles>COc1ccc2c(n1)c(CCNC(=O)c1ccco1)c1n2Cc2cccnc2-1</smiles>

$81 \%$

Fig. 2. Schematic representation of an alternate synthesis of S29434. Note that the yield presented here is not to be taken for the purity of the final compound(s), which was usually above $95 \%$. DMF, dimethylformamide.

(menadione, $125 \mu \mathrm{M}$ ). Naïve Chinese hamster ovary cells (CHO$\mathrm{k} 1-\mathrm{NT}$ ) with basal expression of $\mathrm{QR} 1$ and $\mathrm{QR2}$ and cell lines overexpressing QR1 (CHO-k1-QR1) or overexpressing QR2 (CHO$\mathrm{k} 1-\mathrm{QR} 2$ ) were custom made and purchased from Vectalys (Ramonville, France) (Cassagnes et al., 2015). The experiments were performed on $5.10^{6}$ cells suspended in a final volume of $400 \mu \mathrm{l}$. EPR spectra were obtained at X-band and at room temperature on a Bruker EMX-8/2.7 $(9.86 \mathrm{GHz})$ equipped with a high-sensitivity cavity (4119/HS 0205) and a gaussmeter (Bruker, Wissembourg, France). The analyses were performed with a flat quartz cell FZKI160-5 × $0.3 \mathrm{~mm}$ (Magnettech, Berlin, Germany). WINEPR and SIMFONIA software programs (Bruker) were used for EPR data processing and spectrum computer simulation. Typical scanning parameters were as follows: scan number, 5; modulation amplitude, $1 \mathrm{G}$; modulation frequency, $100 \mathrm{kHz}$; microwave power, $1 \mathrm{~mW}$; sweep width, $100 \mathrm{G}$; sweep time, 41.94 seconds; time constant, $20.48 \mathrm{~ms}$; and magnetic field, 3465-3560 G. The intensities of the EPR signals were evaluated by measuring the amplitude peak to peak of the second line.

\section{Liver and Brain Mitochondrial Respiration}

The animal experimentation component of the studies was conducted with the approval of the local Animal Care and Use Committee and according to the applicable guidelines of the CNRS (http://www.cnrs.fr/ infoslabos/reglementation/euthanasie2.htm). Rats (male, $150 \mathrm{~g}$ ) were sacrificed by cervical dislocation followed by decapitation (fast bleeding). The liver and brain were quickly removed and placed in ice-cold mitochondria isolation buffer $(300 \mathrm{mM}$ sucrose, $10 \mathrm{mM}$ Tris, $1 \mathrm{mM}$
EGTA, pH 7.4). Mitochondria were prepared by differential centrifugation, and the final mitochondrial pellet was resuspended in isolation buffer $(20-80 \mathrm{mg} / \mathrm{ml})$. Mitochondria were resuspended in $5 \mathrm{ml}$ of mitochondrial respiration buffer $[100 \mathrm{mM} \mathrm{KCl}, 40 \mathrm{mM}$ sucrose, $10 \mathrm{mM}$ 2-\{[2-hydroxy-1,1-bis(hydroxymethyl)ethyl]amino\}ethanesulfonic acid, $5 \mathrm{mM} \mathrm{MgCl}_{2}, 1 \mu \mathrm{M}$ EGTA, $5 \mathrm{mM}$ phosphate, $0.4 \%$ fatty acid-free bovine serum albumin, $\mathrm{pH} 7.2$ ), and this suspension was distributed in each of the two chambers (final volume $2 \mathrm{ml}$ ) of the Oxygraph-2k (Oroboros Instruments, Innsbrück, Austria). Temperature was set to $25^{\circ} \mathrm{C}$. Respiration was initiated with addition of glutamate and malate (5 mM each), and a fully stimulated phosphorylating state 3 was obtained by addition of $1.25 \mathrm{mM}$ ADP. Increasing amounts of S29434 were added to one chamber using $1000 \times$ concentrated working solutions in DMSO, and the same volume $(2 \mu \mathrm{l})$ of DMSO was simultaneously added in the other chamber. For the liver experiments, six experiments on three different days with different mitochondria preparations were used. For the brain experiments, three different preparations were used.

\section{Muscle Mitochondrial Respiration}

With the exception of Amplex Red Ultra (Life Technologies, Carlsbad, CA) and BNAH (see earlier discussion), all chemicals and reagents were purchased from Sigma-Aldrich. Male Wistar rats were purchased from Charles River Laboratories, Inc. (Montréal, QC, Canada) and acclimated for approximately 2 weeks in the Animal Care Facility at St. Francis Xavier University (Antigonish, NS, Canada). During the acclimation period, the rats were housed in pairs 
A

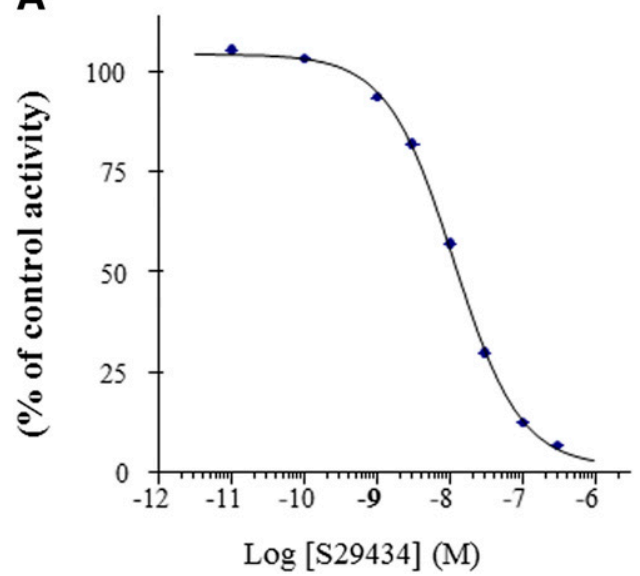

B

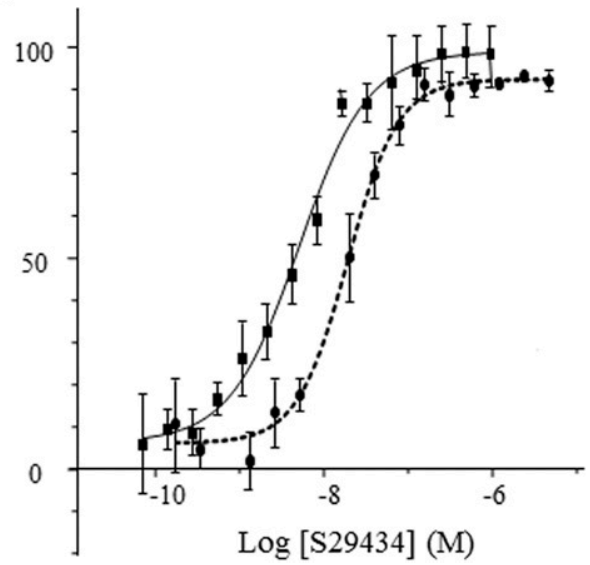

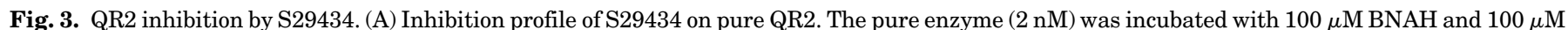

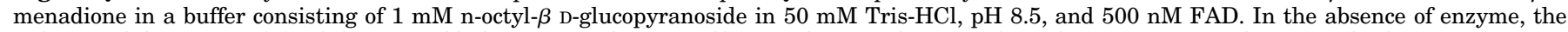

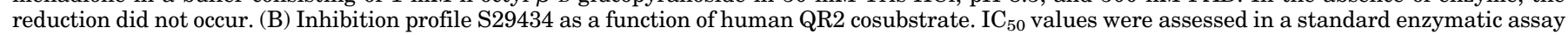

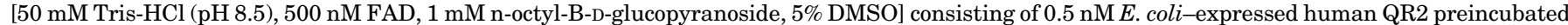

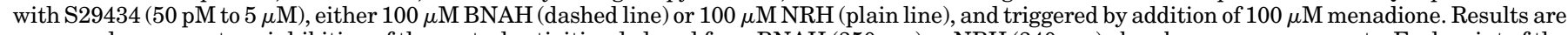

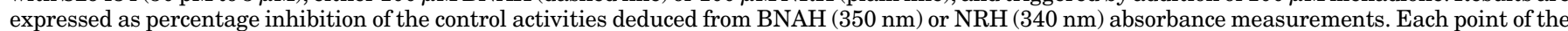
curves represents the mean \pm S.D. of three independent experiments.

in standard cages with a plastic tunnel to provide environmental enrichment. All rats had access to standard rat chow and water ad libitum. The room in which the rats were housed was maintained at $20^{\circ} \mathrm{C} / 22^{\circ} \mathrm{C}$ and $40 \% / 60 \%$ relative humidity and on a reversed 12 -hour light/dark cycle. Approval was granted by the institutional Animal Care Committee at St. Francis Xavier University prior to commencing the study, in accordance with guidelines of the Canadian Council on Animal Care guidelines. Variations of the permeabilized myofiber preparation are published elsewhere (Kuznetsov et al., 2008; Perry et al., 2011, 2013; Pesta and Gnaiger, 2012). In brief, immediately following euthanasia with sodium pentobarbital, two cuts were made on the right gastrocnemius muscle of each animal. Red (i.e., oxidative) portions of the gastrocnemius were extracted and placed in ice-cold buffer X, consisting of $50 \mathrm{mM}$ 4-morpholineethanesulfonic acid, $7.23 \mathrm{mM} \mathrm{K}_{2}$ EGTA, $2.77 \mathrm{mM} \mathrm{CaK}{ }_{2}$ EGTA, $20 \mathrm{mM}$ imidazole, $0.5 \mathrm{mM}$ dithiothreitol, $20 \mathrm{mM}$ taurine, $5.7 \mathrm{mM}$ ATP, $14.3 \mathrm{mM}$ phosphocreatine, and $6.56 \mathrm{mM} \mathrm{MgCl}_{2} \cdot 6 \mathrm{H}_{2} \mathrm{O}$ (pH 7.1). Four fiber bundles from each gastrocnemius section were separated along the longitudinal axis using needle-tipped forceps (Fine Science Tools, Inc., Vancouver, CB, Canada) in ice-cold buffer X under magnification (Discovery V8; Carl Zeiss, Oberkochen, Germany). Following dissection, fiber bundles were placed in vials containing saponin $(50 \mu \mathrm{g} / \mathrm{ml})$ dissolved in $2.0 \mathrm{ml}$ of buffer X. These vials were then placed on a nutating mixer (VWR International, Radnor, PA) and kept at $4^{\circ} \mathrm{C}$ for 30 minutes. After permeabilization, fiber bundles were transferred to a wash buffer solution, consisting of $20 \mathrm{mM}$ taurine, $0.5 \mathrm{mM}$ EGTA, $3 \mathrm{mM} \mathrm{MgCl}_{2}$, $60 \mathrm{mM}$ K-lactobionate, $10 \mathrm{mM} \mathrm{KH} \mathrm{KO}_{4}, 20 \mathrm{mM}$ HEPES, $110 \mathrm{mM}$ sucrose, $20 \mathrm{mM}$ creatine, and $1 \mathrm{~g} / \mathrm{l}$ fatty acid-free bovine serum albumin, $\mathrm{pH}$ 7.1. Fiber bundles remained in the washing buffer (MiR05) for approximately 15 minutes with regular inversion on the nutating mixer until experimentation, after which the samples were placed into the Oxygraph-2k (Oroboros). Blebbistatin was included in the permeabilization buffer, wash buffer, and assay to inhibit spontaneous contraction of muscle fibers (Perry et al., 2011, 2013). Substrate-dependent respiratory oxygen consumption was measured with the Oxygraph-2k (Oroboros). $\mathrm{H}_{2} \mathrm{O}_{2}$ production was monitored in permeabilized rat red gastrocnemius muscle fibers simultaneously with respirometry using an amperometric light-emitting diode module added onto the Oroboros Oxygraph-2k. Prior to the first substrate addition of the respirometric protocol, $10 \mu \mathrm{M}$ Amplex Red Ultra, $5 \mathrm{U} / \mathrm{ml}$ superoxide dismutase, and $1 \mathrm{U} / \mathrm{ml}$ horseradish peroxidase were added to the oxygraph chambers containing the fibers. $\mathrm{H}_{2} \mathrm{O}_{2}$ calibration experiments were performed under the same parameters used for experimental data collection. The relationship between $\mathrm{H}_{2} \mathrm{O}_{2}$ and fluorescence intensity was linear to at least $0.7 \mu \mathrm{M}$. Next, $4 \mathrm{mM}$ malate, $0.2 \mathrm{mM}$ octanoylcarnitine, $5 \mathrm{mM}$ ADP, $20 \mathrm{mM}$ L-lactate, $5 \mathrm{mM}$ $\mathrm{NAD}+, 5 \mathrm{mM}$ pyruvate, and $10 \mu \mathrm{M}$ UK-5099 (2-cyano-3-(1-phenyl-1Hindol-3-yl)-2-propenoic acid) were added in order, and $10 \mathrm{mM}$ glutamate was then added to test glutamate oxidation. Then, $10 \mathrm{mM}$ succinate, $1 \mu \mathrm{M}$ auranofin (thioredoxin reductase inhibitor), and $100 \mu \mathrm{M}$ carmustine (BCNU [1,3-bis(2-chloroethyl)-1-nitrosourea]; inhibitor of thioredoxin and glutathione reductases) were added to the chambers consecutively to inhibit the $\mathrm{H}_{2} \mathrm{O}_{2}$ scavenging system mitochondria. Finally, $\mathrm{H}_{2} \mathrm{O}_{2}$ was titrated to internally calibrate the resorufin signal for $\mathrm{H}_{2} \mathrm{O}_{2}$. Following the conclusion of the respirometry experiments, fibers were placed directly into water to clear out any remaining substrates in the cytosol. After 5 minutes, fiber bundles were then placed in a clean, dry Eppendorf tube and freeze dried using Labconco FreeZone1 (Labconco, Kansas City, MO). Following freeze drying for 5 hours, fiber bundles were weighed on an Excellence Plus XP6 Ultra-Microbalance (Mettler Toledo, Columbus, OH).

Results represent means \pm S.D. $(n=4)$. A two-way, repeatedmeasures analysis of variance (ANOVA) was completed for each oxygen flux and hydrogen peroxide production to identify any interaction among the three groups of exercise and histamine receptor antagonists, exercise only, and control. This step was followed by a one-way ANOVA with repeated measures (Bonferroni post hoc analysis) to examine differences between different substrate additions. Data analysis was completed using Prism 7 (GraphPad). The $\alpha$ level for statistical significance was set at 0.05 .

\section{Measurement of Mitochondrial ROS Levels by Flow Cytometry in Cells}

MitoSox (Thermo Fisher Scientific Life Technologies, Molecular Probes, Waltham, MA) was used to measure mitochondrial superoxide levels in cells by flow cytometry. HepG2 were a kind gift from Dr. Eugenio Arcidiacono (University Magna Graecia, Catanzaro, Italy) and were cultured in high-glucose (4.5 g/l) Dulbecco's modified essential medium (DMEM) as previously described (Lascala et al., 2018). U373 cells were cultured in low-glucose DMEM. Both types of DMEM were supplemented with $10 \%$ fetal bovine serum, $1 \%$ penicillin and streptomycin solution (Pen-Strep), and 1\% glutamine, all from Carlo Erba srl (Milan, Italy). HepG2 and U373 cells were seeded on 
24-well plates at densities of $80 \times 10^{3}$ and $60 \times 10^{3}$ per well, respectively, 2 days before starting treatments. Cells were exposed to S29434 or vehicle (DMSO) for 6, 18, and 24 hours before the end of the experiment. Next, the cells were washed with prewarmed serumfree DMEM (high and low glucose, depending on the cell type) and then incubated with MitoSox (2.5 $\mu \mathrm{M}, 15$ minutes) diluted in DMEM as described earlier. The experiment was finished by washing the cells twice with prewarmed phosphate-buffer saline followed by trypsinmediated detachment of cells and flow cytometry analysis according to previously published protocols (Janda et al., 2013).

\section{Transient Silencing of QR2 Expression and Autophagy Assay in Hepatic Cells}

HepG2 cells were cultured as described earlier. Cells were seeded on 12-well plates 1 day before transfection at a density of $30,000 \mathrm{~cm}^{-2}$. Nonsilencing or control small interfering RNA (siRNA; AllStars) and a set of human QR2-targeting siRNA were purchased from Qiagen (Hilden, Germany). Transfection of HepG2 cells was performed using Lipofectamine 2000 (Life Technologies, Invitrogen, Monza MB, Italy) and Optimem (Life Technologies, Invitrogen) according to the manufacturer's instructions. In brief, 360 pmol siRNA QR2 or control siRNA and $18 \mu \mathrm{l}$ of Lipofectamine 2000 were mixed and incubated in $600 \mu \mathrm{l}$ of Optimem for 15-20 minutes at room temperature. Each well containing HepG2 cells in $500 \mu \mathrm{l}$ of serum-free medium was overlaid with $100 \mu \mathrm{l}$ of siRNA-Lipofectamine complexes and incubated in a cellculture incubator. After 5 hours, the medium was changed for DMEM supplemented with fetal bovine serum without Pen-Strep. Twentyfour hours later, the cells were treated with S29434 or DMSO for 24 hours in regular medium with Pen-Strep. Chloroquine $(25 \mu \mathrm{M})$ was added 3 hours before the end of the experiment to half of the samples. The cells were lysed, and the lysates were run on $12 \%$ SDS-PAGE gels and assayed for LC3 and QR2 expression by western blotting, as previously described (Janda et al., 2015). The antibodies were as follows: polyclonal rabbit anti-LC3A/B (MBL International Co., Woburn, MA) used at 1:2000, anti-glyceraldehyde-3-phosphate dehydrogenase (Santa Cruz Biotech, Dallas, TX) used at 1:500, and antiQR2 (Sigma-Aldrich) used at 1:1000.

\section{Testing S29434 against Neuronal Degeneration In Vitro}

Animals were housed, handled, and taken care of in accordance with recommendations of the Guide for the Care and Use of Laboratory Animals of the National Institutes of Health (National Institutes of Health publication number 85-23, revised 1996) and the European Union Council Directives (2010/63/EU) (http://eur-lex.europa. eu/LexUriServ/LexUriServ.do?uri=OJ:L:2010:276:0033:0079:FR:PDF). Experimental procedures were authorized by the ethical committee on animal experiments (Comité Charles Darwin \#5). Cultures were prepared from the ventral midbrain of Wistar rat embryos at gestational age 15.5 days (Janvier LABORATORIES, Le Genest-St.-Isle, France). Dissociated cells in suspension obtained by mechanical trituration of midbrain tissue pieces were seeded at a density of $1.2-1.5 \times 10^{5}$ cells $/ \mathrm{cm}^{2}$ onto Nunc 48 -well multidish plates (ThermoFisher Scientific, Waltham, MA) precoated with $1 \mathrm{mg} / \mathrm{ml}$ polyethylenimine diluted in borate buffer, $\mathrm{pH}$ 8.3, as previously described (Toulorge et al., 2011). In some experiments, the cultures were maintained in N5 medium supplemented with $5 \mathrm{mM}$ glucose, $5 \%$ horse serum, and $0.5 \%$ fetal calf serum, except for the first 3 days in vitro, when the concentration of fetal calf serum was set at $2.5 \%$ to favor initial culture maturation (Guerreiro et al., 2008). In the other experiments, we used a chemically defined serum-free medium consisting of equal volumes of Dulbecco's minimal essential medium and Ham's F12 nutrient mixture (Life Technologies, Invitrogen) supplemented with $10 \mu \mathrm{g} / \mathrm{ml}$ insulin, $30 \mathrm{mM}$ glucose, and 100 IU/ml Pen-Strep (Troadec et al., 2001). Dopamine neurons were detected by tyrosine hydroxylase immunofluorescence staining using procedures previously described (Toulorge et al., 2011). These neurons represented approximately $2 \%$ to $3 \%$ of the total number of neuronal cells present in these cultures after plating. The cultures, fixed for 12 minutes using $4 \%$ formaldehyde in Dulbecco's PBS, were washed twice with PBS before an incubation step at $4^{\circ} \mathrm{C}$ for $24-72$ hours with primary antibodies. A monoclonal antityrosine hydroxylase antibody diluted 1/5000 (ImmunoStar, Inc., Hudson, WI) or a polyclonal antityrosine hydroxylase antibody diluted 1/1000 (US Biologicals, Salem, MA) was used to assess survival of dopamine neurons. Cell counting was performed with a Nikon TE 2000 inverted microscope (Nikon, Champigny-sur-Marne, France) at $200 \times$ magnification, using a $20 \times$ objective matched with a $10 \times$ ocular. The number of tyrosine hydroxylase + neurons in each culture well was estimated after counting visual fields distributed along the $x$ - and $y$-axes. The functional integrity and synaptic function of dopamine neurons were evaluated by their ability to accumulate $\left[{ }^{3} \mathrm{H}\right]$-dopamine $(50 \mathrm{nM}, 40 \mathrm{Ci} / \mathrm{mmol}$; PerkinElmer, Courtaboeuf, France), as previously described (Guerreiro et al., 2008). When using N5 medium supplemented with serum, dopamine neurons in culture degenerate spontaneously and progressively during the maturation process of these cultures (Toulorge et al., 2011). Thus, in this setting, pharmacological treatments were initiated immediately after plating and were then renewed daily after replacing a fraction (twothirds) of culture medium. Dopamine cell survival was assessed in 9-day in vitro cultures, i.e., at a stage where about $70 \%$ of dopamine neurons have already died (Toulorge et al., 2011). Some sets of cultures were treated with veratridine $(0.8 \mu \mathrm{M})$, a depolarizing agent used as a positive control for neuroprotection in this setting (Salthun-Lassalle et al., 2004). Treatments with the mitochondrial toxin 1-methyl-4-phenylpyridinium $\left(\mathrm{MPP}^{+}\right)$were performed in cultures in which the spontaneous death process was prevented by supplementing the N5 medium with a depolarizing concentration of $\mathrm{K}^{+}(30 \mathrm{mM})$ in the presence of $1 \mu \mathrm{M}$ $N$-methyl-D-aspartate receptor antagonist dizocilpine ([5R,10S]-[1]5-methyl-10,11-dihydro-5H-dibenzo[a,d]cyclohepten-5,10-imine,

MK-801), as previously described (Salthun-Lassalle et al., 2004). Note that this treatment did not interfere with the neurotoxic effects of $\mathrm{MPP}^{+} . \mathrm{MPP}^{+}$was applied to midbrain cultures between 4 and 6 days in culture, whereas protective treatments were added after 2 days of in vitro culture and renewed thereafter until fixation of the cultures at 6 days of in culture. Finally, oxidative stress-mediated dopamine cell death was achieved by placing the cultures in a defined serum-free DMEM/Ham's F12 nutrient mixture medium containing $1.5 \mu \mathrm{M}$ ferrous iron (Troadec et al., 2001). The antimitotic compound cytarabine $(1.5 \mu \mathrm{M})$ was added to the cultures after plating to prevent glial cell proliferation. Dopamine cell survival was assessed at 5 days in culture in these conditions. Experimental values expressed as the mean \pm S.E.M. were derived from triplicates of three independent experiments. Data were analyzed using the SigmaPlot 12.5 software (Systat Software Inc., San Jose, CA) with one-way ANOVA followed by the StudentNewman-Keuls post hoc test for all pairwise comparisons.

\section{Object Recognition in Mice}

Experiments were performed in a quiet, dimly lit room in a dark Plexiglas chamber $(\mathrm{L} \times \mathrm{W} \times \mathrm{H}: 25 \times 35 \times 25 \mathrm{~cm})$. On the day before the test day (day 0 , familiarization phase), the animals were allowed to explore the test apparatus for 2.5 minutes. Twenty-four hours later (day 1, acquisition phase), after a 30 -minute pretreatment time after drug treatment ( 1 and $15 \mathrm{mg} / \mathrm{kg}$, i.p.), each test animal was placed in the middle of the test box from the previous day, and the 5-minute acquisition trial began. In the first trial, mice were allowed to explore two identical objects (10 seconds per object within a total 5-minute period). During test time, the exploration time (ET) was measured with a stopwatch. ET is defined as direct, active olfactory exploration of objects 1 and 2 . In general, it consists of nosing and sniffing of the edge and the top, as well as approaching and crossing the line approximately $1 \mathrm{~cm}$ around the objects. Posturing and mounting are not included in measures of investigation. On day 2 (24 hours later, retention phase), a new object and a familiar object were placed in the test box, and the animals were replaced in the box for 4 minutes and ET measured again. The discrimination index was calculated as: 
[exploration time of new object in seconds $(\mathrm{N})$ - exploration time of familiar object in seconds $(\mathrm{F})] /[$ exploration time of new object in seconds $(\mathrm{N})+$ familiar object exploration time in seconds $(\mathrm{F})]=$ $(\mathrm{N}-\mathrm{F}) /(\mathrm{N}+\mathrm{F})$.

A thorough description of these methods can be found in previous publications (Ennaceur and Delacour, 1988; Bartolini et al., 1996; Bevins and Besheer, 2006). For in vivo data, one-way ANOVA was performed (followed by Dunnett's multiple comparisons test) for the discrimination index data.

\section{Results}

Chemistry. S29434 was synthesized by Guillaumet and colleagues in the late $1990 \mathrm{~s}$ as a compound to explore the melatoninergic system(s). We characterized it as an interesting tool for QR2 studies after we discovered that MT3, the unconventional melatonin binding site we and others have described (Duncan et al., 1988; Paul et al., 1999), was indeed in QR2 (Nosjean et al., 2000). The first synthesis, as is typical, aimed at producing several examples of tetracyclic chemicals that could be assimilated into two indolic structures fused together. The goal at that stage was to document the chemical series, of which S29434 was the most potent. The route led to tens of milligrams of material and was not meant to yield greater quantity. The synthetic route is summarized in Fig. 1. The second synthesis scheme was intended to rationalize the yields of each step, even if alternative synthetic steps had to be substituted in the original protocols, as summarized in Fig. 2. This alternative route was meant to deliver gram amounts of the compound. Full details of the synthetic routes are given as supplemental material. Crucially, the final product, used in most of the experiments presented here, was $99 \%$ pure, based on liquid chromatography-mass spectrometry and NMR spectra data (Supplemental Materials). The mass spectrometry data as well as the NMR data confirmed the structure of the final product.

Inhibition of QR2. Various reports have shown that, depending on the substrate/cosubstrate couples used to measure the catalytic activity of $\mathrm{QR} 2$, the recorded $\mathrm{IC}_{50}$ values range from 1 to $40 \mathrm{nM}$ (Table 1). We stabilized our system in a standard configuration (see Materials and Methods) for all internal needs for screening of this enzyme (S29434 was the reference compound) and identified an $\mathrm{IC}_{50}$ of $16 \pm 3.2 \mathrm{nM}$ (mean \pm S.D., $n=51$ ). The considerable amount of data accumulated on this material render this information quite robust, at least when using the human cloned enzyme BNAH as cosubstrate and menadione as substrate. A typical sigmoid curve of the concentration/activity relationship is presented in Fig. 3A. We also present a comparison of S29434 inhibition of the enzyme catalytic activity while using BNAH, a synthetic cosubstrate, or NRH, a natural candidate cosubstrate of QR2 (Fig. 3B). With the natural cosubstrate, the inhibition curve is shifted slightly leftward, from an $\mathrm{IC}_{50}$ of $17 \pm 3 \mathrm{nM}$ in the presence of BNAH to $5 \pm 1 \mathrm{nM}$ with $\mathrm{NRH}$ (mean \pm S.D.). Nevertheless, the use of BNAH is recommended because the compound is commercially available and slightly more robust than its ribosylated counterpart (Boutin et al., 2005). S29434 is a competitive inhibitor, as reported previously (Mailliet et al., 2005; Ferry et al., 2010; Pegan et al., 2011).

Inhibitor Specificity: Standard Panel of Molecular Targets (Eurofins-CEREP Profiles). We first checked the specificity of the inhibitor S29434 for possible off-target activities (from the QR2 perspective). First, we assessed its activity in a series of standard assays designed to evaluate its behavior against a selection of receptors and enzymes [see, for example, Millan et al. (2012)]. All tests were performed in duplicate, independently, at two concentrations: $100 \mathrm{nM}$ and $10 \mu \mathrm{M}$. With one exception, the results indicated no capacity of S29434 to inhibit binding at or activity of the selected targets, even at high concentrations ( $>10 \mu \mathrm{M})$ (data not shown). The notable exception was that S29434 could displace binding of 2-iodomelatonin at the melatonin receptor MT2, showing an affinity of $0.14 \mu \mathrm{M}$. This result is not surprising given that S29434 was initially synthesized as part of a melatonin receptor agonist discovery process. Finally, S29434 showed no cytotoxic activities toward several standard cancer cell lines (Caco-2, HCT116, HT-29, and L1210) up to $10 \mathrm{mM}$, based on MTT assays (Berridge et al., 2005).

Inhibitor Specificity: Kinases. Duncan et al. (2008) reported the toxicity of two casein kinase 2 inhibitors, 4,5,6,7-1H-tetrabromobenzimidazole and 2-dimethylamino4,5,6,7-tetrabromo- $1 H$-benzimidazole, presumably because of their inhibition of QR2 catalytic activity. Similarly, Rix et al. (2007) reported that QR2 is the only nonkinase target of the BCR-ABL inhibitors imatinib and nilotinib. Thus, we assessed whether the QR2 inhibitor could inhibit one or several catalytic activities of a panel of kinases. Of the 46 included kinases, no inhibition greater than $20 \%$ was recorded for S29434 at $100 \mathrm{nM}$ (data not shown). Because of the high potency of S29434, no higher concentrations were tested.

TABLE 1

Summary of the $\mathrm{IC}_{50}$ values from different laboratories and conditions with S29434

\begin{tabular}{|c|c|c|c|c|c|}
\hline Enzyme & Cosubstrate & Substrate & Signal & $\mathrm{IC}_{50}($ mean \pm S.D. $)$ & References \\
\hline \multicolumn{6}{|c|}{$n M$} \\
\hline hQR2 expressed in $E$. coli & BNAH $(100 \mu \mathrm{M})$ & Menadione & Abs $350 \mathrm{~nm}$ & $16 \pm 3.2$ & Present paper \\
\hline hQR2 expressed in $E$. coli & NRH $(100 \mu \mathrm{M})$ & Menadione & Abs $340 \mathrm{~nm}$ & $5 \pm 1$ & Present paper \\
\hline hQR2 expressed in $E$. coli & BNAH $(100 \mu \mathrm{M})$ & Menadione & Abs $350 \mathrm{~nm}$ & $17 \pm 3$ & Present paper \\
\hline hQR2 expressed in CHO & BNAH $(100 \mu \mathrm{M})$ & Menadione & Fluo BNAH & $14 \pm 1$ & Mailliet et al. (2005) \\
\hline hQR2 expressed in $\mathrm{CHO}$ & BNAH $(100 \mu \mathrm{M})$ & Menadione & Fluo BNAH & $14 \pm 7$ & Pegan et al. (2011) \\
\hline hQR2 expressed in $E$. coli & $\mathrm{NMeH}(100 \mu \mathrm{M})$ & Menadione & Abs $360 \mathrm{~nm}$ & $2.4 \pm 0.7$ & Pegan et al. (2011) \\
\hline hQR2 expressed in $E$. coli & $\mathrm{NMeH}(100 \mu \mathrm{M})$ & Formosan & Abs $612 \mathrm{~nm}^{a}$ & $11 \pm 2$ & Pegan et al. (2011) \\
\hline $\mathrm{hQR} 2$ expressed in insect cells & BNAH $(100 \mu \mathrm{M})$ & Menadione & Fluo BNAH & $6.7 \pm 0.7$ & Ferry et al. (2010) \\
\hline hQR2 expressed in insect cells & NRH $(100 \mu \mathrm{M})$ & Menadione & Fluo NRH & $0.7 \pm 0.1$ & Ferry et al. (2010) \\
\hline hQR2 expressed in insect cells & NRH $(100 \mu \mathrm{M})$ & Coenzyme Q2 & Fluo NRH & $5 \pm 0.9$ & Ferry et al. (2010) \\
\hline
\end{tabular}

Abs, absorbance assay; CHO, Chinese hamster ovary; Fluo, fluorimetric assay; hQR2, human QR2.

${ }^{a}$ Absorbance of MTT(3-(4,5-dimethylthiazol-2-yl)-2,5-diphenyltetrazolium bromide). 
Inhibitor Specificity: QR1. The best candidate for possible specificity of S29434 would be QR1, the closest relative of QR2. We also wanted to confirm that dicoumarol, the standard QR1 inhibitor (Ernster et al., 1962) in wide use, could not inhibit QR2. Based on previous catalytic activity measurement comparisons between the two enzymes (Ferry et al., 2010), S29434 showed good selectivity for QR2 over QR1, with the reverse result for dicoumarol. In a new set of experiments (Fig. 4), we used EPR analysis and showed that S29434 at $10 \mu \mathrm{M}$ did not inhibit QR1 compared with full inhibition by dicoumarol. Indeed, S29434 clearly had no effect on the intensity of the EPR spectrum, as represented by the sixlined spectrum characteristic of the radical adduct [DMPO$\mathrm{CH} 3] \cdot$. This radical adduct originates from the spin trapping of $\cdot \mathrm{CH} 3$ formed by reaction of $\cdot \mathrm{OH}$ (produced during the reoxidation of menadiol) with DMSO.

Inhibitor Specificity: Sirtuin. Because of the link between sirtuin-mediated NAD degradation (Jiang et al., 2017) and QR2's use of a possible NADH catabolite, we sought to ensure that the inhibitor's activity did not lead to upstream inhibition of sirtuin, diminishing the availability of its cosubstrate. Even at $10 \mu \mathrm{M}, \mathrm{S} 29434$ did not inhibit sirtuin, whereas superoxide dismutase and diphenyleneiodonium chloride were potent inhibitors (not shown).

Inhibitor Specificity: NADPH Oxidase. In addition to mitochondria, a main cellular source of ROS production is NADPH oxidase (Sorce et al., 2017; Teixeira et al., 2017). Thus, we checked the capacity of S29434 to inhibit this family of enzymes, using the NADPH oxidase inhibitor diphenyleneiodonium. The reference compound led to a complete inhibition, but S29434 remained poorly active up to $10 \mu \mathrm{M}$, at which point it began to inhibit about $20 \%$ of ROS production from those cells (Supplemental Fig. 3).

S29434/Quinone Reductase 2 Characteristics: Binding Kinetics. Resveratrol has been described as a potent inhibitor of QR2 (Buryanovskyy et al., 2004) and thus might serve as a reference compound in some conditions. The binding of either S29434 or resveratrol leads to quenching of the intrinsic fluorescence of QR2 (oxidized FAD). We used preequilibrium stopped-flow spectroscopy to measure the realtime binding kinetics of the two compounds and compare their binding mechanisms (Fig. 5). Resveratrol binding kinetics were too fast at $25^{\circ} \mathrm{C}$, so we decreased the temperature to $4^{\circ} \mathrm{C}$ to slow the rate. The results were consistent with a single-step binding mechanism, governed by an association rate constant $\left(k_{1}\right)$ of $1910^{6} \mathrm{~s}^{-1} \mathrm{M}^{-1}$ and a dissociation rate constant $\left(k_{-1}\right)$ of 27 seconds $^{-1}$. A K $\mathrm{D}_{\mathrm{D}}$ estimate of $1.4 \mu \mathrm{M}$ was calculated from the ratio $k_{-1} / k_{1}$, which is in good agreement with the isothermal titration calorimetric data obtained on the same enzyme batch, yielding a $\mathrm{K}_{\mathrm{D}}$ of $0.6 \mu \mathrm{M}$ at $25^{\circ} \mathrm{C}$ (data not shown). In contrast, S29434 displayed slower but biphasic binding transients consistent with a two-step binding mechanism involving a conformational change. Replotting the $k_{\text {obs }}$ rate constants for the fast phase yielded a linear plot, diagnostic of a bimolecular association step, governed by a $k_{1}$ of $1410^{6} \mathrm{~s}^{-1}$ $\mathrm{M}^{-1}$ and a dissociation rate constant $\left(k_{-1}\right)$ of 61 seconds ${ }^{-1} \cdot \mathrm{M}^{-1}$. A $K_{D}$ estimate for this encounter complex of $4.4 \mu \mathrm{M}$ can be calculated from the ratio $k_{-1} / k_{1}$. The observed rate of the slow phase was independent of S29434 concentration in the investigated range, staying close to 30 seconds $^{-1}$. Therefore, this step likely reflects a conformational rearrangement, either following binding (induced fit) or preceding it (conformational selection). These preliminary kinetic data strongly suggest the evolution of the binding mechanism from the low-affinity single-step binder resveratrol to the high-affinity two-step binder S29434. They also are in agreement with results from our mass spectrometry studies of the inhibitor/enzyme binding (Antoine et al., 2012).
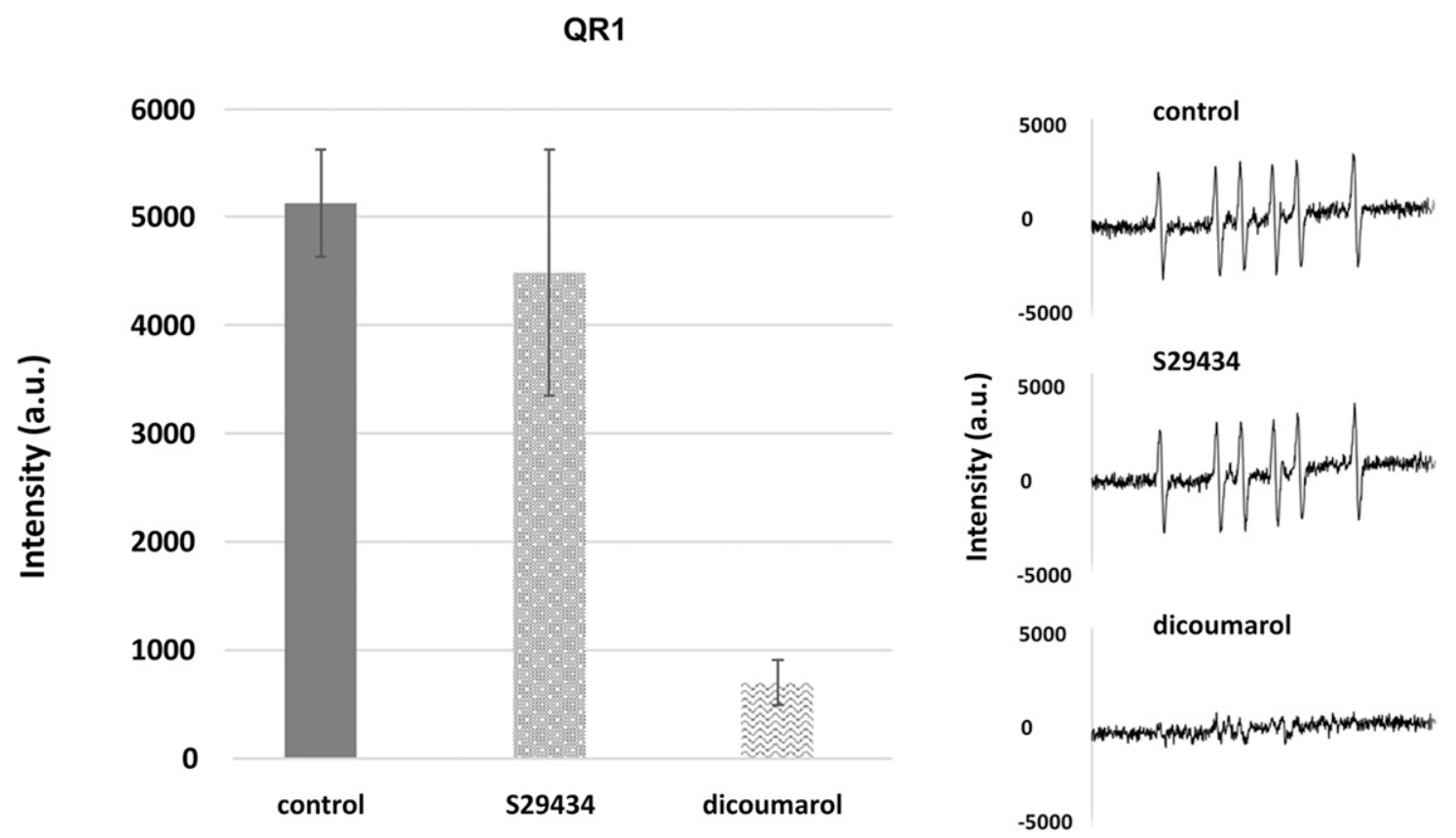

Fig. 4. Specificity of S29434 toward QR1. Comparison with dicoumarol. ROS production recorded with purified QR1 (5 $\mu \mathrm{g} / \mathrm{ml})$ in the presence of NADH $(100 \mu \mathrm{M})$ and menadione $(100 \mu \mathrm{M})$ following preincubation with buffer (control), S29434 (10 $\mu \mathrm{M})$, or dicoumarol (10 $\mu \mathrm{M})$. The corresponding EPR spectra are presented in the right panels. The production of ROS was evaluated by the double integration of the EPR spectra obtained with DMPO (50 mM) as a spin trap. Histograms represent the intensity of the signals as the mean \pm S.D. of three independent measures. a.u., arbitrary units. 

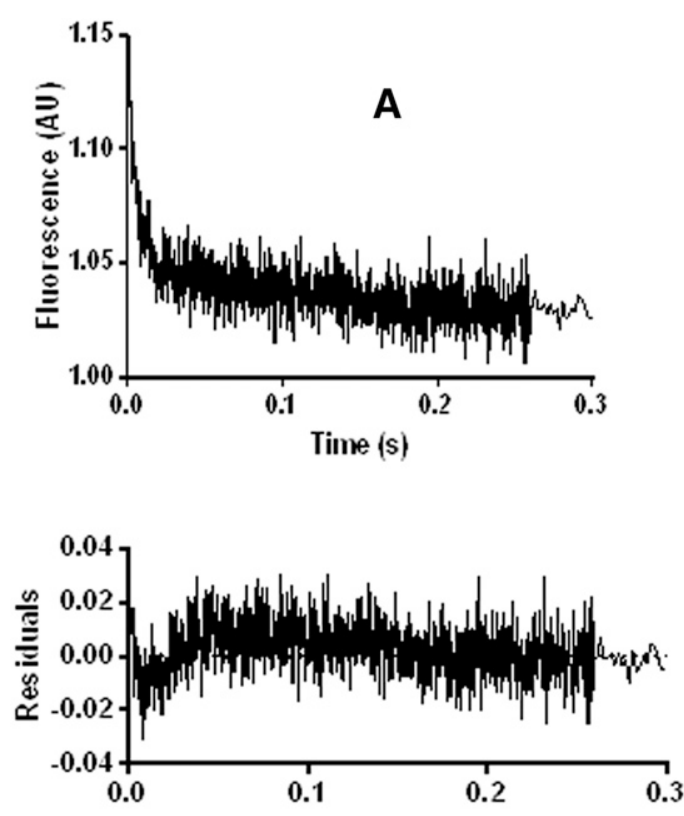

B

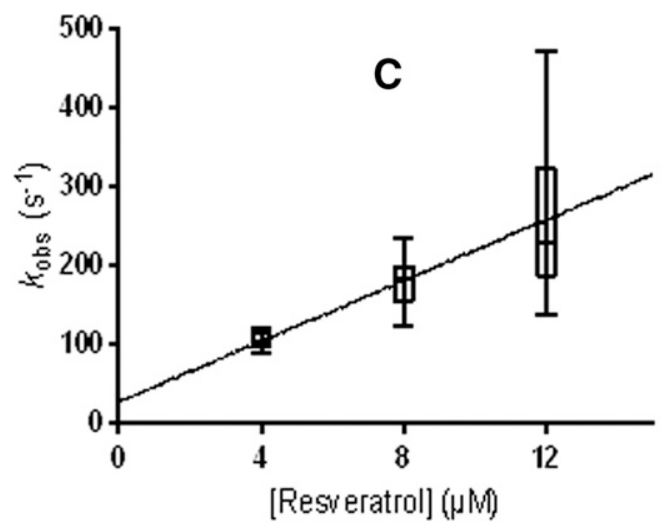

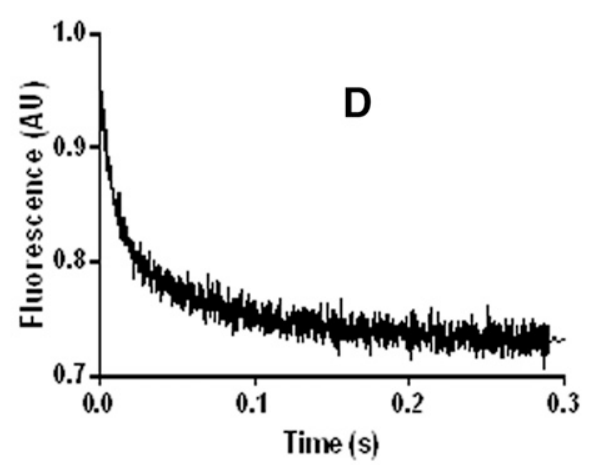

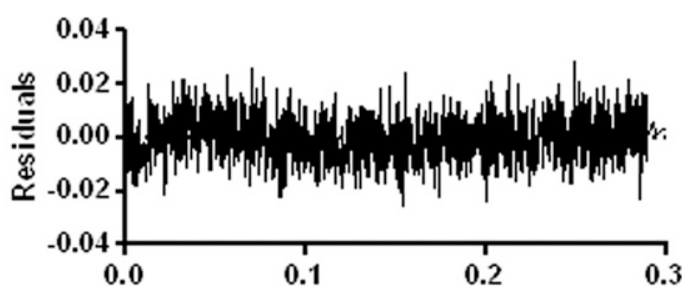

E

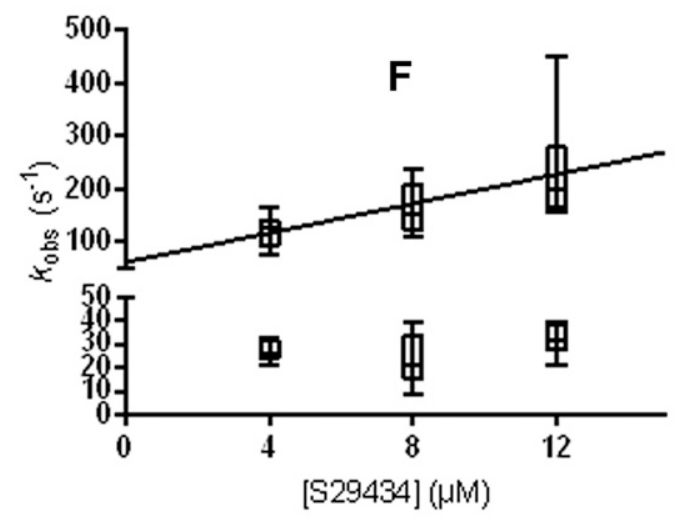

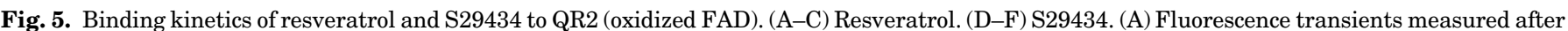

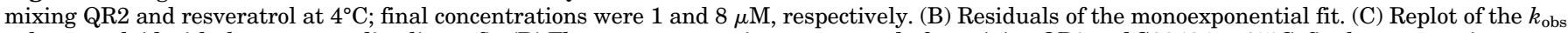

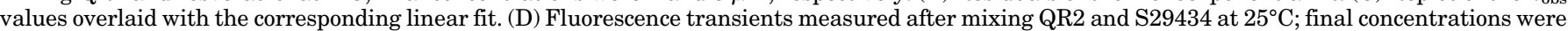

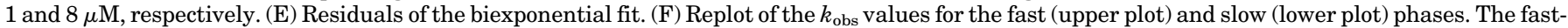

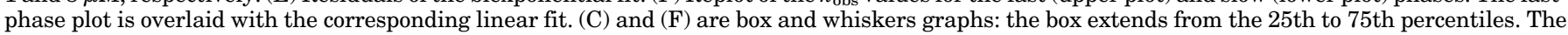

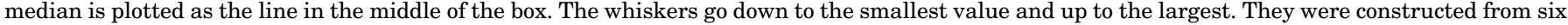
replicates. AU, arbitrary units.

S29434/QR2 Characteristics: Cocrystallization. Through various collaborations, we generated cocrystals of S29434 in QR2 that we deposited in the Protein Data Bank (www.rcsb.org) (Pegan et al., 2011; Gerard et al., 2018), including several unpublished crystals at $1.4-\AA$ resolution (4QOD;); however, the interactions and orientation of the compound in the crystal were not discussed in great detail. In brief, as shown in Fig. 6, the ligand S29434 fits deeply into a QR2 hydrophobic cavity (Protein Data Bank: 3OX3), interacting with subunits A and B with its amido-furan side chains pointing toward the solvent. It makes $\pi$-edge interactions with Trp105 and Phe126, $\pi-\pi$ interactions with Phe178, and a water-mediated H-bond with Asn161. Additional $\pi-\pi$ interactions exist with cofactor FAD. Although the furan moiety makes an additional hydrophobic interaction with Ile194 from QR2, this part of the molecule also seems to hang out of the catalytic site. This feature potentially offers the possibility of modification of this part of the molecule with, for instance, a fluorophore moiety, producing associated properties, as has been done for chromen-2-one derivatives as inhibitors of QR1 (Bian et al., 2017).

Transit of S29434 across the Cellular Membrane and Blood-Brain Barrier. We next conducted a series of experiments to characterize the properties of S29434 in terms of pharmacokinetics and cellular penetration. First, we measured the behavior of S29434 in an in cellulo blood-brain barrier model (Cecchelli et al., 1999) based on cocultures of bovine brain microvessel endothelial cells and rat astrocytes (Booher and Sensenbrenner, 1972) showing expression of the P-glycoprotein 1 efflux transporter (pGP). For this purpose, we measured the permeability surface total (filter + collagen + 


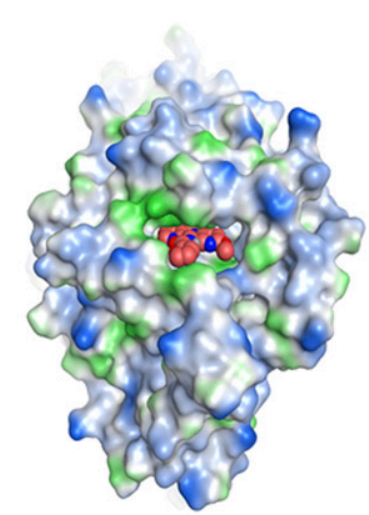

A

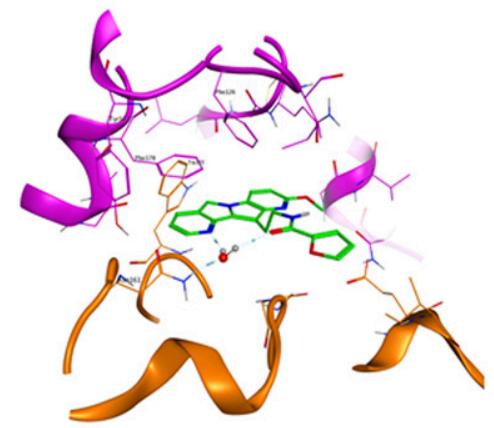

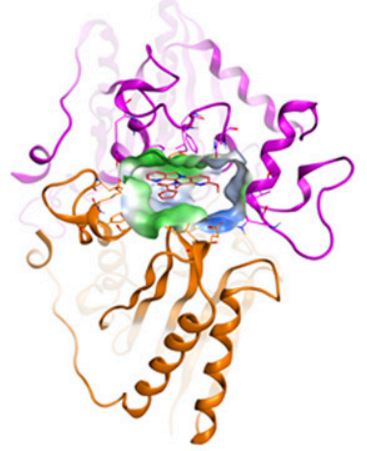

B

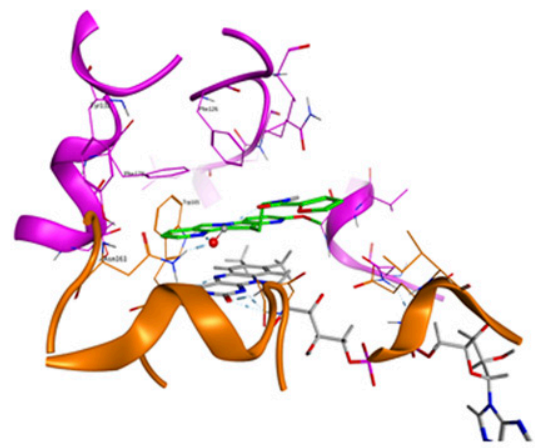

Fig. 6. Cocrystallization of S29434 with QR2. This cocrystallization of human QR2 with S29434 has been deposited in the Protein Data Bank under the Protein Data Bank code 3OX3 (Pegan et al., 2011). (A) QR2 molecular surface colored by lipophilicity (blue, hydrophilic regions; green, hydrophobic regions). Ligand S29434 (coral, spacefilling model). (B) Ligand S29434 binding cavity (orange, chain 1; pink, chain B; coral, ligand; lipophilic surface). (C) Left: QR2/S29434 interactions; right: QR2/S29434 interactions with FAD (gray).

\section{C}

cells) and the permeability surface filter (filter + collagen without cells) expressed in milliliters per minute. These values were 4.58 and $3.78 \mathrm{~cm} / \mathrm{min}$, respectively. Therefore, the permeability surface total/permeability surface filter (permeability class) ratio for S29434 was determined to be $121 \%$, showing no barrier effect of the blood-brain barrier (BBB) cells

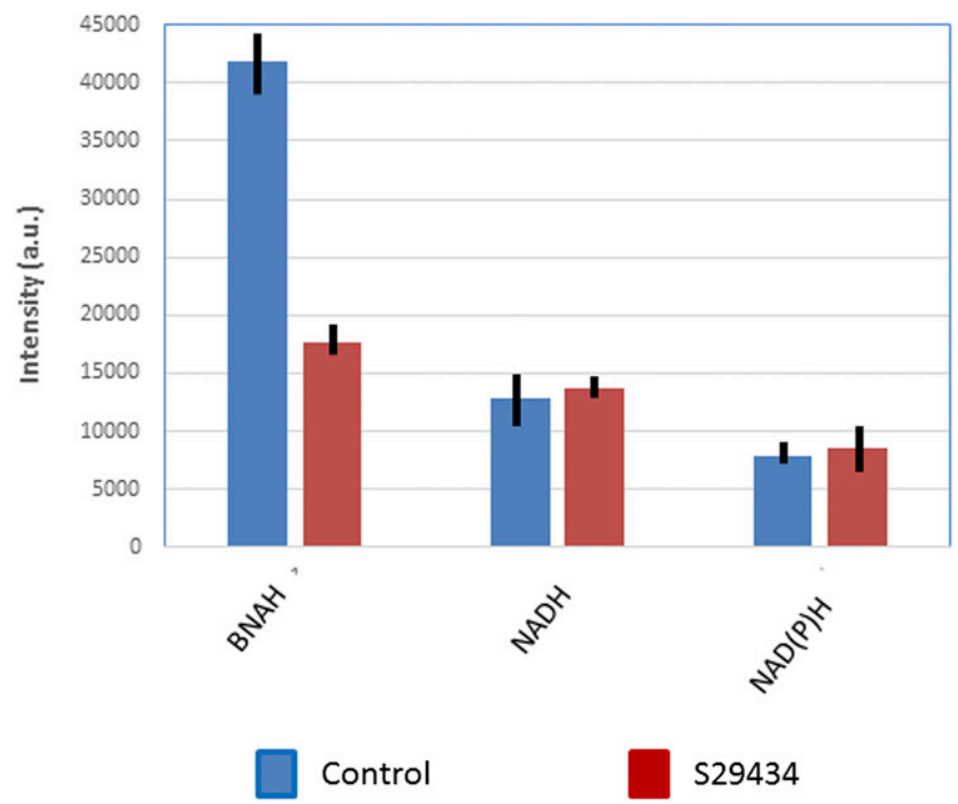

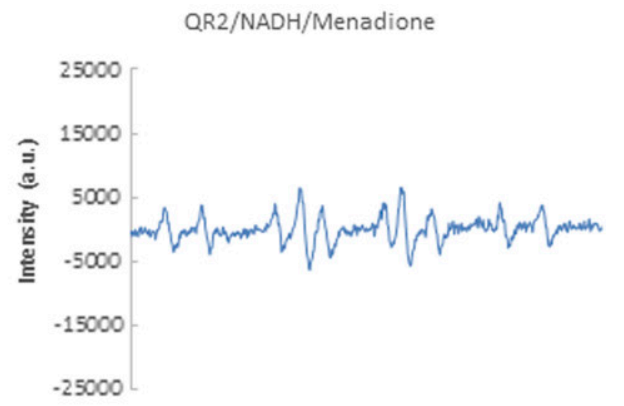

QR2/BNAH/Menadione

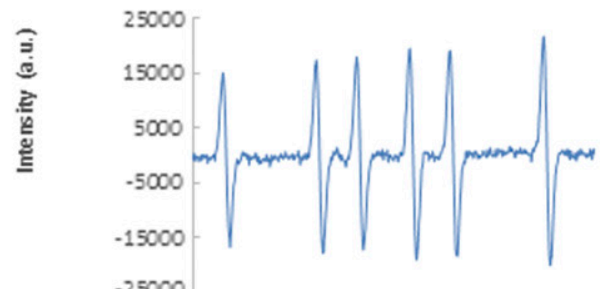

Fig. 7. Effect of cosubstrate and inhibitor on the QR2-dependent production of ROS. (Left panel) ROS production recorded with purified QR2 (25.6 $\mu \mathrm{g} / \mathrm{ml}$ ) and different cosubstrates [BNAH, NADH, or NAD $(\mathrm{P}) \mathrm{H}]$ in the presence or absence of $20 \mu \mathrm{M}$ S29434. ROS production was evaluated by the double integration of the EPR spectra obtained with DMPO $(50 \mathrm{mM})$ as a spin trap. The histograms represent the mean \pm S.D. of at least four independent measures. (Right panel) A representative crude EPR spectrum recorded with BNAH or NADH and menadione is shown. a.u., arbitrary units. 
compared with filter + collagen alone, classifying S29434 as a highly permeable compound (Cecchelli et al., 1999). Second, S29434 was tested on the well known Caco2 model, expressing the main efflux transporters present at the intestinal and bloodbrain barriers (pGP, ATP-binding cassette super-family G member 2 [BCRP] and multidrug resistance-associated protein 2 [MRP2]). The apical-to-basolateral apparent permeability was $3310^{-6} \mathrm{~cm} / \mathrm{s}$ (incubation concentration at $20 \mu \mathrm{M}$ ), also corresponding to a highly permeable compound, and was in the same range as that of propranolol, the highly passive permeability reference. The basolateral-to-apical permeability was not quantified in these experiments, but the high apical-tobasolateral permeability suggested no efflux transporter involvement.

QR2 and ROS Production. QR2 activity can be followed by EPR (Reybier et al., 2011; Cassagnes et al., 2015). As noted, this functional assay is indirectly linked to the enzyme. Indeed, the reaction product, a quinol, has poor stability in standard conditions. In the presence of oxygen, the quinol is oxidized back into the original quinone, which in turn serves as substrate for the enzyme. The result is a futile cycle between the two chemical species with the concomitant production of ROS [see Reybier et al. (2011) for a complete discussion]. This process seems to occur in acellular as well as cellular contexts (Cassagnes et al., 2017). Here, we completed and supported these findings, showing by EPR spectroscopy that pure QR2 in the presence of its cosubstrate (BNAH) produces ROS during catalytic activity (Fig. 7); S29434 inhibits this effect. Furthermore, under those experimental conditions, neither $\mathrm{NADH}$ nor $\mathrm{NAD}(\mathrm{P}) \mathrm{H}$ could substitute for BNAH. Under basal conditions (no substrate or cosubstrate of QR2), very low levels of ROS were recorded.

QR2 and ROS Production: Mitochondrial Respiration in Liver and Brain. Because S29434 diminishes the ROS produced during the futile cycle between quinol and quinone in aerobic conditions (Reybier et al., 2011), we wanted to ensure that this effect did not result from direct interference of S29434 with ROS even though the compound is inactive in other ROS-generating systems (such as mitochondria). Our results confirmed that $\mathrm{S} 29434$ cannot trap hydroxyl radicals $\left({ }^{\circ} \mathrm{OH}\right)$ produced via the Fenton reaction or superoxide radicals $\left(\mathrm{O}_{2}^{\circ}\right)$ produced from crown ether and $\mathrm{KO}_{2}$ (not shown). We also tested another source of cellular ROS production: the mitochondria. To evaluate the mitochondrial effect of the drugs, mitochondrial respiration was settled with complex I substrates (glutamate/malate) and in phosphorylating conditions (1.25 mM ADP). The relative effect of increasing amounts of S29434 is indicated by the ratio of respiratory rate compound/solvent, with a value of 1 indicating a lack of effect. With this approach, S29434 produced no effect up to $10 \mu \mathrm{M}$ concentrations and revealed no statistically significant changes in respiratory rate with rat liver or rat brain mitochondria (Fig. 8). The system was validated with experiments with rotenone or oligomycin, two known inhibitors of the mitochondrial respiratory chain, with results similar to those reported in the literature (data not shown).

QR2 and ROS Production: Mitochondrial Respiration in Muscle. The next step involved independent assessment of mitochondria in saponin-permeabilized rat gastrocnemius muscle fibers. As illustrated in Fig. 9A, the conditions resulted in no differences in respiration, confirming that neither BNAH nor S29434 had an effect in these samples. Likewise, BNAH and $\mathrm{S} 29434$ did not affect $\mathrm{H}_{2} \mathrm{O}_{2}$ production (Fig. 9B). Expression of $\mathrm{H}_{2} \mathrm{O}_{2}$ as a percentage of $\mathrm{O}_{2}$ flux yielded a statistically significant difference between $\mathrm{BNAH}$ and BNAH + S29434 (Fig. 9C) in the absence of ADP only. This finding suggested that $\mathrm{BNAH}$ may increase mitochondrial $\mathrm{H}_{2} \mathrm{O}_{2}$ in nonphosphorylating (i.e., state 4) conditions. However, because neither respiration nor $\mathrm{H}_{2} \mathrm{O}_{2}$ alone was affected, a more plausible explanation is that the difference in proportional $\mathrm{H}_{2} \mathrm{O}_{2}$ production is the result of nonmitochondrial sources of $\mathrm{H}_{2} \mathrm{O}_{2}$ in the samples and not of bulk $\mathrm{O}_{2}$ flux.

Effects of $\mathbf{S 2 9 4 3 4}$ on Cells: Nervous System Cells. S29434 was described as having a beneficial effect against paraquat-induced toxicity in cellulo and in vivo (Janda et al., 2013, 2015), suggesting a potential role of QR2 in Parkinson's

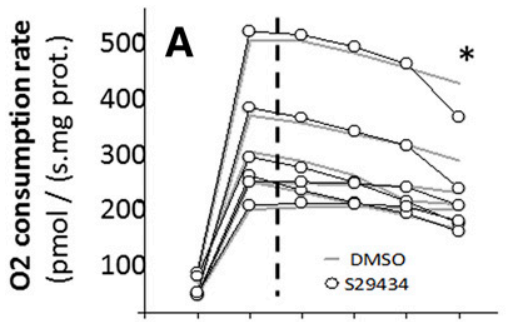

GMADP $10^{-7} 10^{-6} 10^{-5} 10^{-4}$

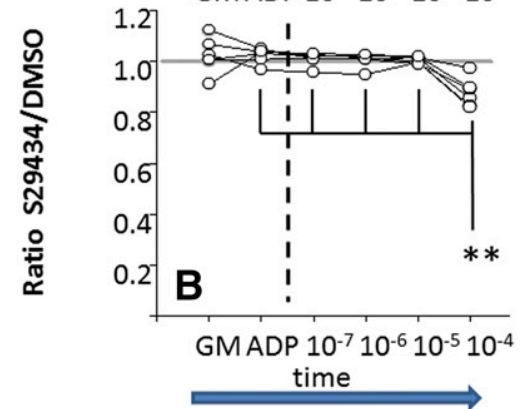

Successive additions to the mitochondrial suspension
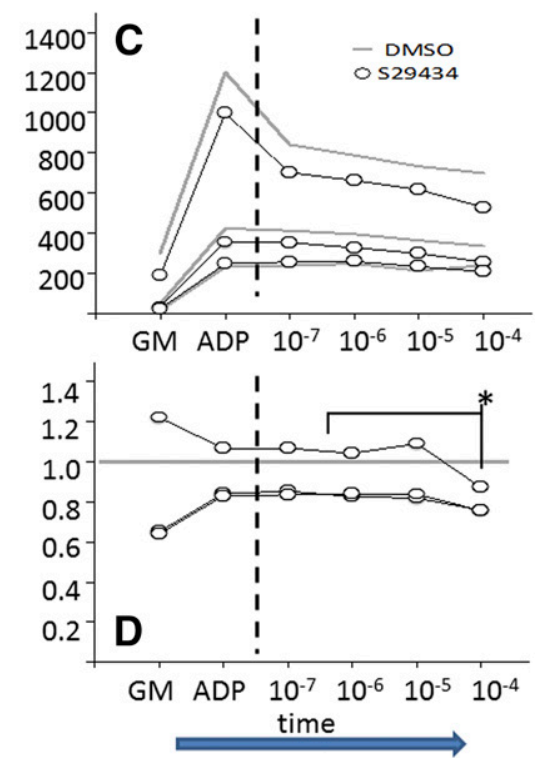

Fig. 8. Effect of S29434 on respiration of rat liver and brain mitochondria. Rat liver mitochondria (A and $\mathrm{B}$ ) and rat brain mitochondria (C and $\mathrm{D})$ oxygen consumption was measured simultaneously in the two chambers of the respirometer Oxygraph-2k (www.oroboros.at). Successive additions are indicated below the $x$-axis (time). First (left to the vertical dotted line), we settled a fast respiring phosphorylating state by addition of substrates glutamate/malate (GM) and ADP. Then (right to the dotted line), four additions of increasing concentrations $\left(10^{-7}-10^{-4} \mathrm{M}\right)$ of S29434 were made (black line and empty dots) as a control, and the same volumes of solvent (DMSO) were added (gray line). (A and B) Mean value of the oxygen consumption rate $[$ in $\mathrm{pmol} /(\mathrm{s} \cdot \mathrm{mg}$ protein)]. $* P<0.05$ with a paired $t$ test to compare the oxygen consumption rate with S29434 $(100 \mu \mathrm{M})$ or solvent for the same period of time. Experiments were run six times on different rat liver preparations. (C and D) Ratio between the rates for S29434/solvent. $* * P<0.01$ with the Mann-Whitney rank test (http://vassarstats.net/) for the comparison of the ratio observed with S29434 at $10^{-4}$ with each of the other comparable situations (ADP and no further addition, S29434 $\left.10^{-7}-10^{-5} \mathrm{M}\right)$. Experiments were run three times on different rat brain preparations. 

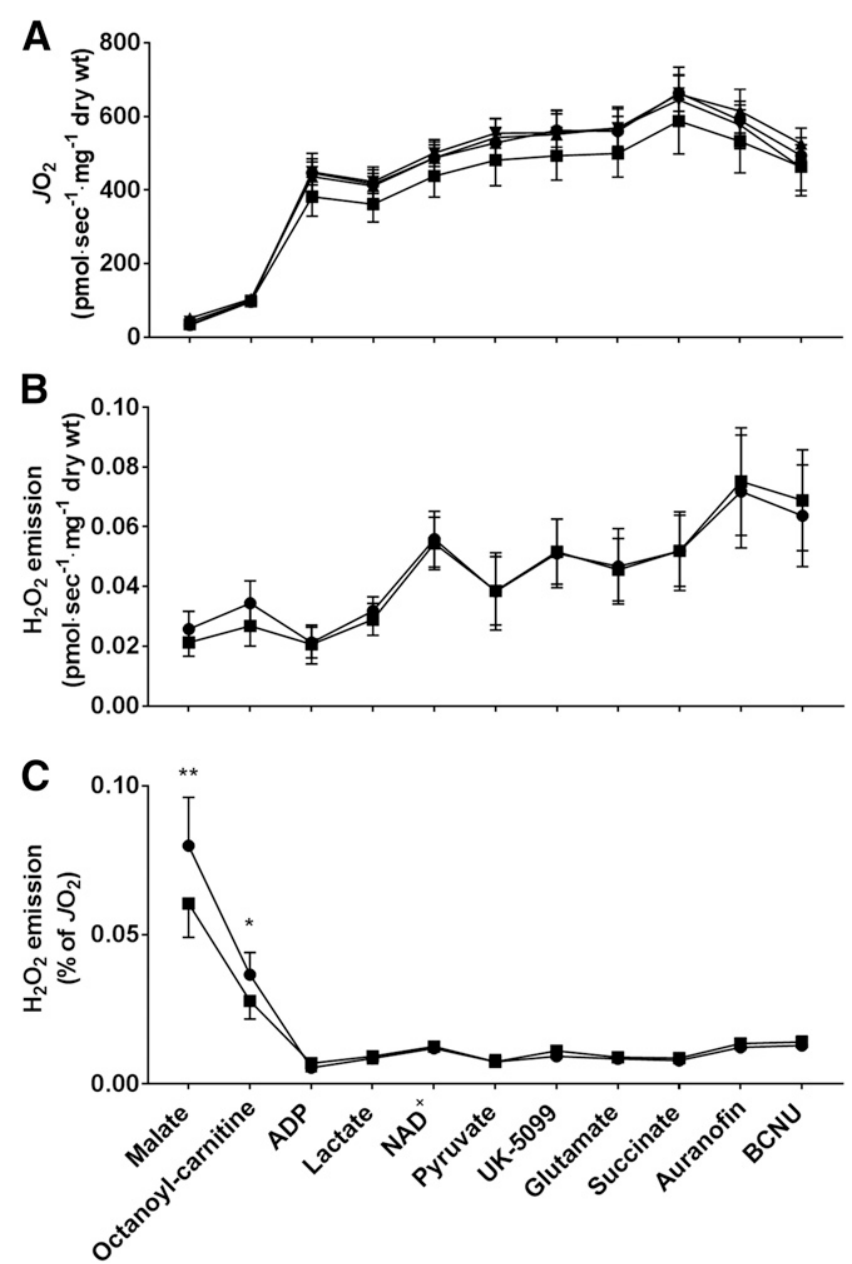

Fig. 9. Effects of $\mathrm{S} 29434$ on mitochondrial respiration or $\mathrm{H}_{2} \mathrm{O}_{2}$ production in permeabilized skeletal muscle. Saponin-permeabilized rat red gastrocnemius skeletal muscle fibers were incubated in the following prior to assay: $100 \mu \mathrm{M}$ BNAH (circles), $30 \mu \mathrm{M}$ S29434 (downward triangles), $100 \mu \mathrm{M}$ BNAH $+30 \mu \mathrm{M}$ S29434 (squares), or control (vehicle: $18.75 \mu \mathrm{l}$ of DMSO, upward triangles). Subsequently, respiration was measured in all conditions (A), and $\mathrm{H}_{2} \mathrm{O}_{2}$ in $\mathrm{S} 29434$ and $\mathrm{BNAH}+\mathrm{S} 29434$ conditions (B). $\mathrm{H}_{2} \mathrm{O}_{2}$ as a percentage of $\mathrm{O}_{2}$ consumed was also calculated (C). Results represent means \pm S.D.; $n=4$. ${ }^{*} P<0.05$; $* * P<0.01, n=7$. BCNU, 1,3-bis (2-chloroethyl)-1-nitrosourea.

disease. This finding prompted us to analyze its effect in models of neuronal degeneration in cellulo. Primary midbrain dopamine neurons in culture were established from fetal rat ventral midbrains. In this system, S29434 (up $100 \mu \mathrm{M}$ ) did not reduce spontaneous loss of dopamine neurons in culture (Fig. $10 \mathrm{~A})$, while the reference neuroprotective molecule, veratridine, efficiently rescued these cells. S29434 also failed to protect dopaminergic cells from iron-induced oxidative stress (with iron in the culture medium) caused by a Fenton-type reaction occurring spontaneously (Fig. 10B). At $100 \mu \mathrm{M}$, S29434 even tended to become slightly toxic. As expected, however, the chelation of iron with deferoxamine $(10 \mu \mathrm{M})$ led to robust protection under these conditions. In contrast to these observations, S29434 showed concentration-dependent protective effects against the mitochondrial complex I inhibitor $\mathrm{MPP}^{+}$(Fig. 10C). This effect was already present at 0.1 $\mu \mathrm{M}$, peaked at $1 \mu \mathrm{M}$, and declined progressively between 10 and $100 \mu \mathrm{M}$. Nicotine, used at $10 \mu \mathrm{M}$ as a positive control, also rescued dopamine cells. Because $\mathrm{MPP}^{+}$is selectively taken up by the dopamine transporter to exert its selective toxicity toward dopamine neurons, we tested whether S29434 operated as an inhibitor of dopamine uptake. We thus performed this routine experiment according to details already published in our laboratory (Guerreiro et al., 2008). S29434 did not bind to the ad hoc transporter (not shown), a finding that strongly suggests that it blocks the intrinsic mechanism of $\mathrm{MPP}^{+}$toxicity, not its transport.

Effects of S29434 on Cells: Autophagy. Autophagy is a vesicle-mediated pathway that ensures recognition and transport of defective organelles and protein aggregates to lysosomes, where they are digested. We tested if S29434 could induce autophagy in human HepG2 cells, a cell line derived from liver, the organ with the highest level of QR2 expression (Nosjean et al., 2000). S29434 dose-dependently induced LC3II, a marker of autophagy vesicles (Fig. 11A). Silencing of QR2 by more than 50\% increased basal LC3-II levels and suppressed LC3-II induction by S29434. This result indicated that the autophagy response to this compound does not depend on its off-target effects but is directly mediated by QR2 inhibition (second panel in Fig. 11, A and B). Of importance, LC3-II was further accumulated in S29434-treated cells when autophagy flux was blocked by the lysosomal inhibitor CLQ, indicating true induction of autophagy by the QR2 inhibitor (Fig. 11B). Further details are given in Supplemental Data 8, including discussion of the whole blots. These data confirm that QR2 plays a role in regulation of autophagy. Oxidative stress and redox reactions have a strong impact on autophagy machinery (Janda et al., 2012, 2015). However, because QR2 inhibition does not reduce basal ROS levels in HepG2 cells (Fig. 12A) or U373 astrocytes (Fig. 12B), the mechanism by which S29434 triggers autophagy should be mitochondrial ROS-independent and QR2-dependent, as suggested by the aforementioned results (Fig. 11, A and B; Supplemental Data 8).

Effects of S29434 In Vivo: Object-Recognition Tests in CH3 Wild-Type Mice. An interplay between QR2 and memory has been reported (Brouillette and Quirion, 2008; Benoit et al., 2010; Rappaport et al., 2015). To further assess this relationship, we used the inhibitor S29434 in the objectrecognition test. The test trial with the new object was carried out 24 hours after the acquisition trial with two identical objects. S29434 was given intraperitoneally 30 minutes before the acquisition trial ( 1 and $15 \mathrm{mg} / \mathrm{kg}$, respectively). Figure 13A shows the discrimination index, where $\mathrm{N}$ is the time spent inspecting the new object and $\mathrm{F}$ is the time spent exploring the familiar object during the test trial. Both concentrations of S29434 led to an enhanced time of exploration of the unfamiliar object, although the effects of the two doses ( 1 and $15 \mathrm{mg} / \mathrm{kg}$ ) did not differ. Under these circumstances and experimental conditions, the object-recognition memory of wild-type mice was enhanced by a factor of $\sim 16$ by intraperitoneal injection of S29434 over the performance of control mice in the same test. As reported for other models (Rappaport et al., 2015), this finding suggests that by inhibiting QR2, S29434 has a positive effect at least on this particular aspect of memory.

In Vivo Considerations on S29434: Object-Recognition Memory in the Knockout CH3QR2 ${ }^{-/-}$Mice. The same experiments were carried out on knockout (KO) $\mathrm{CH} 3 \mathrm{QR2} 2^{-1-}$ mice, and the results are presented in Fig. 13B. For QR2 KO animals, the results strongly reinforce the previous findings. Indeed, treated ( 1 or $15 \mathrm{mg} / \mathrm{kg}$ ) and control animals did not 
A

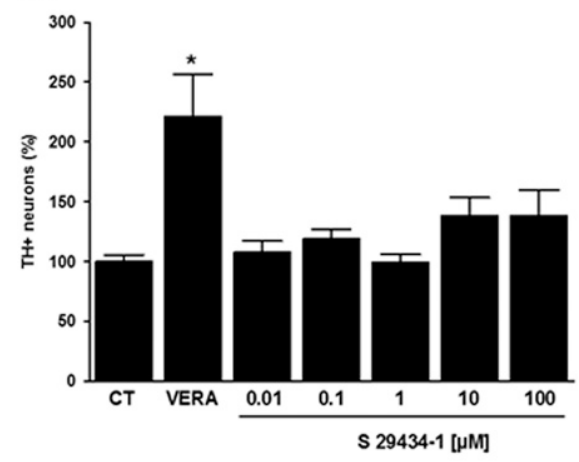

B

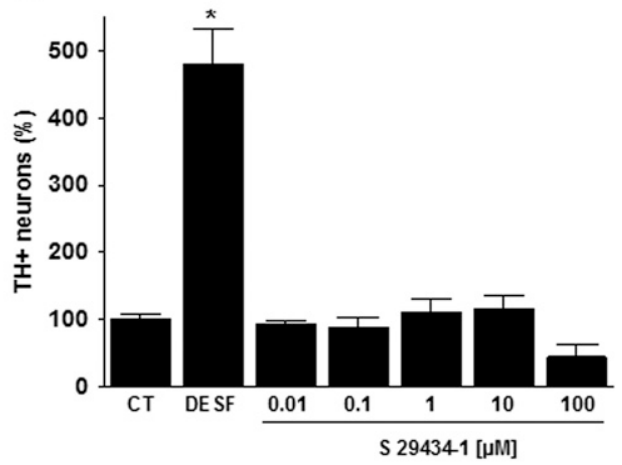

Fig. 10. S29434 effects on neuronal cell cultures. (A) S29434 does not protect dopamine neurons that degenerate spontaneously in midbrain cultures maintained with serum-supplemented N5 medium. The reference compound is veratridine (VERA; $0.8 \mu \mathrm{M}$ ). ${ }^{*} P<0.05$ vs corresponding controls. (B) S29434 does not protect dopamine neurons that degenerate from oxidative stress in midbrain cultures maintained with a chemically defined medium containing catalytic iron. The reference compound was deferoxamine (DESF; $10 \mu \mathrm{M}$ ). ${ }^{*} P<0.05$ vs. corresponding controls. (C) S29434 protects dopamine neurons against $\mathrm{MPP}^{+}$-induced cell death. Survival of tyrosine hydroxylase-positive neurons in midbrain cultures treated (open bars) or not treated with $\mathrm{MPP}^{+}$at a concentration of $3 \mu \mathrm{M}$. Reference compound is nicotine (Nic; $10 \mu \mathrm{M})$. $* P<0.05$ vs. corresponding controls. In all experiments, S29434 was tested at concentrations ranging from $10 \mathrm{nM}$ to $100 \mu \mathrm{M}$, as indicated above the black line on figures A, B, \& C. CT, controls. differ, indicating that the product has no further effect on this parameter. Because the QR2 KO animals were genetically deficient in this enzyme only (Mailliet et al., 2004), the lack of S29434 activity strongly suggests that the compound is specific to QR2. Furthermore, we know that these animals did not lose their learning capacity compared with the wild-type mice, as demonstrated in several tests, including the Morris water maze, object recognition, and rotarod performance test. They performed better than their wild-type counterparts on all of these tests (Benoit et al., 2010).

\section{Discussion}

General. Molecular pharmacology is based on either the manipulation (including the mutagenesis) of targets or their inhibition/activation by synthetic or natural chemicals. A good example is certainly dicoumarol, the QR1 reference inhibitor. This compound has been widely used in the literature (over 2700 references), yet many of its off-target effects bias the interpretation of the data obtained [see discussion by Scott et al. (2011)]. To avoid a similar scenario with S29434, we sought to fill in information gaps related to this compound, including assessments of its in cellulo and in vivo activity, stability, permeability, and overall specificity and to publish a clear synthesis method.

Chemistry. S29434 belongs to a family of flat tetracyclic compounds, some that we (Boussard et al., 2006) and others have described, e.g., cryptolepines (Onyeibor et al., 2005; Lavrado et al., 2008; Whittell et al., 2011) or other types of indolizino-indolones (Bhattacharya et al., 2001). Compounds that inhibit QR2 activity have also been reported, including pyrroloquinoline ammosamides (Reddy et al., 2012), indolequinones

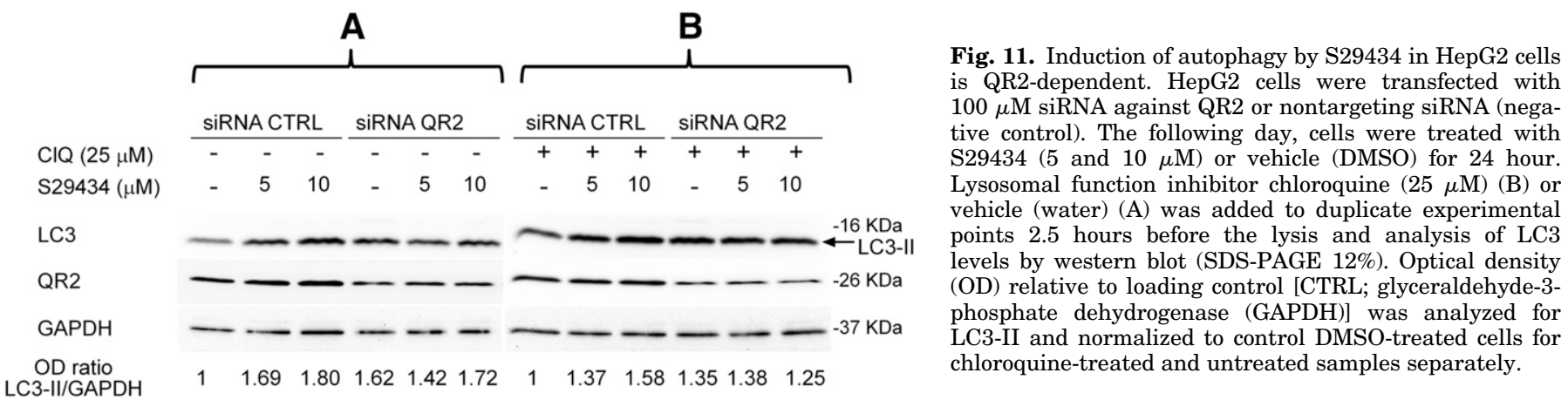

Fig. 11. Induction of autophagy by S29434 in HepG2 cells tive control). The following day, cells were treated with S29434 (5 and $10 \mu \mathrm{M}$ ) or vehicle (DMSO) for 24 hour. Lysosomal function inhibitor chloroquine $(25 \mu \mathrm{M})(\mathrm{B})$ or vehicle (water) (A) was added to duplicate experimental points 2.5 hours before the lysis and analysis of LC3 levels by western blot (SDS-PAGE 12\%). Optical density (OD) relative to loading control [CTRL; glyceraldehyde-3(O) chloroquine-treated and untreated samples separately. 

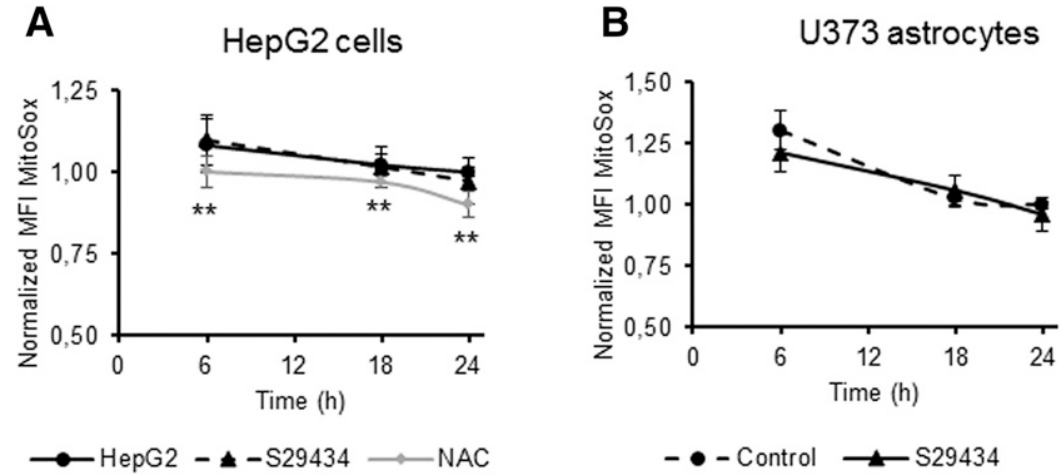

Fig. 12. S29434 has no effect on basal mitochondrial ROS levels in cells. Subconfluent human hepatoma (HepG2) (A) and astrocytoma (U373) (B) cell lines were treated with S29434 $(10 \mu \mathrm{M}), N$-acetylcysteine $(5 \mathrm{mM})$, or vehicle (DMSO) for 6, 18, and 24 hours before analysis. ROS levels were determined by flow cytometry after staining with MitoSOX. The graphs show the mean \pm S.E.M. from three independent experiments performed at least in triplicate. Statistical analysis two-way ANOVA followed by Tukey's post-test, time versus pharmacological treatment, three groups per three times, $n=3$ or 4 samples in each group, $* * P<0.01$ when compared with control at the same time point. MFI, mean fluorescence intensity.
(Dufour et al., 2011; Yan et al., 2011), and furan-amidines (Alnabulsi et al., 2018). Because few, if any, have been characterized beyond their enzymatic test activities, we believe that the present report is noteworthy because it describes S29434 activity in different in cellulo and in vivo situations.

Acellular Behavior: Specificity. In an acellular system, S29434 is a potent inhibitor of QR2 activity $\left(\mathrm{IC}_{50}=\sim 10 \mathrm{nM}\right)$. It marginally inhibits QR1, if at all. Furthermore, S29434 does not potently inhibit more than 100 other targets, with the sole exception of the melatonin receptor 2 , for which it has a binding affinity in the micromolar range. Of course, the notion of specificity is limited to targets for which measures are available. It is not possible to know if S29434 will uniquely recognize QR2 among the protein products of the 26,000 genes in the human genome. The mechanism by which S29434 binds to and interacts with QR2 has been described (Pegan et al., 2011; Antoine et al., 2012). Here, we add information about the way the compound occupies the catalytic site of the enzyme. We also identify ways to measure S29434's affinity for QR2 in complement with its capacity to inhibit it. These two notions (affinity for the enzyme and inhibition potency) are too often conflated, and it is important to distinguish them. Furthermore, the biophysical measure allowed us to evaluate how long the compound sits in the catalytic site, which can vary widely across a series of analog compounds for a given enzyme, ranging from seconds to hours.

Stability In Vivo: Membrane Crossing. Here, S29434 was demonstrated to be highly permeable on both an in vitro BBB model that expresses the pGP and on the Caco2 model expressing the three main efflux transporters present at both intestinal and BBB barriers (pGP, BCRP, MRP2); this suggests no efflux involvement, even if not directly demonstrated by bidirectional experiments. It was already clear that S29434 could penetrate different types of primary or cultivated cells (Benoit et al., 2010; Janda et al., 2013; Chen et al., 2017). Even if an in vivo pharmacokinetic study would have helped to determine the pharmacokinetic parameters, such as T1/2 or bioavailability, the fact that the compound was active in rodent models when administered by intraperitoneal route (Janda et al., 2015; Rappaport et al., 2015) demonstrated that at least sufficient in vivo brain exposures (level and duration) were reached in mammals. Nevertheless, it is clear for us that a complete assessment of the plasma stability as well as a quantification of the part of the injected molecule that reaches the brain of an animal after intraperitoneal treatment would greatly help the future studies.

Cellular Aspects: ROS. As noted, QR2 is closely linked with ROS production. Thus, our main goal was to ensure that

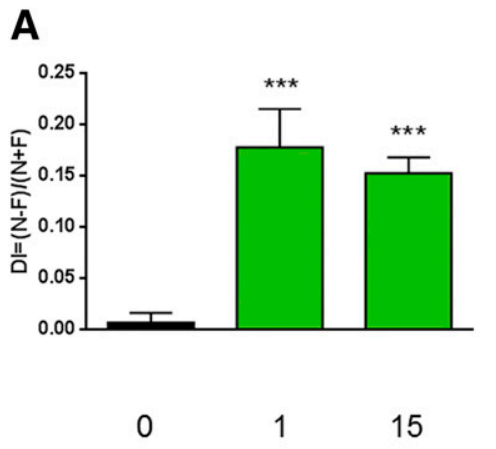

B

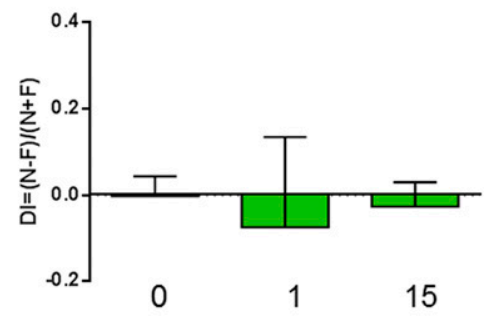

S29434 i.p. treatment $(\mathrm{mg} / \mathrm{kg})$

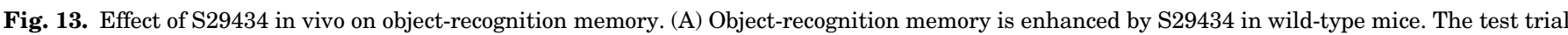
with the new object was carried out 24 hours after the acquisition trial with two identical objects. S29434 was given intraperitoneally 30 minutes before the acquisition trial ( 1 and $15 \mathrm{mg} / \mathrm{kg}$ ). The discrimination index (DI) is shown, where $\mathrm{N}$ is the time spent inspecting the new object and $\mathrm{F}$ is the time spent exploring the familiar object during the test trial. Data are the mean \pm S.E.M., $n=9$ to 10 /group. $* * * P<0.001$, one-way ANOVA followed by Dunnett's test versus medium-treated control animals $(0 \mathrm{mg} / \mathrm{kg})$. (B) Lack of effect of S29434 on object-recognition memory in CH3QR2 ${ }^{-/-}$mice. The test trial with the new object was carried out 24 hours after the acquisition trial with two identical objects. S29434 was given intraperitoneally 30 minutes before the acquisition trial ( 1 and $15 \mathrm{mg} / \mathrm{kg}$ ). The DI is shown, where $\mathrm{N}$ is the time spent inspecting the new object and $\mathrm{F}$ is the time spent exploring the familiar object during the test trial. Data are the mean \pm S.E.M., $n=10$ group. 
S29434 was not a scavenger per se, and that the reduction in ROS bursts in cellular experiments was the result of S29434 interference with QR2. Indeed, the results showed that S29434 can inhibit QR2-dependent production of ROS in various cell types, including hepatoma HepG2 cells, the astroglial cell line U373 (Janda et al., 2013), and primary dopaminergic neurons (Cassagnes et al., 2017). However, it does not alter basal cellular ROS levels when applied in the absence of exogenous QR2 activators. For example, we treated HepG2 and U373 cells with S29434 at 6, 18, and 24 hours before measuring intracellular ROS levels with MitoSox, a fluorescent compound recognizing preferentially mitochondrial superoxide anion (Polster et al., 2014). MitoSox fluorescence was unaltered by S29434 at all experimental times in HepG2, but a nonspecific antioxidant, $N$-acetyl cysteine, statistically significantly reduced basal ROS levels (Fig. 12). Mitochondria are the main source of cellular ROS in physiologic conditions. Thus, we conducted detailed studies on mitochondria from various organs to validate that in all conditions, S29434 did not inhibit this ROS production. Furthermore, S29434 did not inhibit NADPH oxidase or impair ROS produced from a Fenton-type reaction that exerted toxicity neuronal cells. These data support that S29434 is specific to QR2.

Cellular Aspects: Autophagy. The autophagy pathway mitigates cellular damage from stress and toxic agents and plays an important role in ensuring basal protein and organelle turnover (Janda et al., 2012; Dagda et al., 2013). While studying the antioxidant effects of S29434 on paraquatinduced toxicity, we discovered that this compound has yet another property: the ability to restore basal autophagy in paraquat-treated astrocytes. In addition, S29434 induces autophagy in the absence of paraquat, a response mediated by a QR2-dependent mechanism in astrocytes (Janda et al., 2015). The data presented here indicate that the QR2dependent mechanism of autophagy induction can be generalized to other cell types, such as liver cells. Furthermore, we showed that this effect does not correlate with any detectable modulation of ROS levels in liver cells, thus excluding a possible ROS-dependent basis of the proautophagic activity of S29434. The accumulation of LC3-II in the presence of CLQ is believed to result from a blockade of lysosome activity. It might depend in part, however, on the ability of the antimalaria drug CLQ to bind and inhibit QR2 directly, although with poor affinity compared with S29434 (Kwiek et al., 2004; Leung and Shilton, 2015). Indeed, the direct effect of CLQ on QR2 is much weaker than its inhibitory effect on lysosomal digestion because LC3-II still accumulates when QR2 expression is reduced by siRNA (Fig. 11B, compare lines 1 and 4).

Cellular Aspects: Neuronal Cells. $\mathrm{MPP}^{+}$, the active metabolite of 1-methyl-4-phényl-1,2,3,6-tetrahydropyridine, inhibits mitochondrial complex I. When delivered into mice, $\mathrm{MPP}^{+}$ leads to a Parkinson-like syndrome. We know that QR2 does not recognize 1-methyl-4-phényl-1,2,3,6-tetrahydropyridine as a substrate; nevertheless, S29434 shows concentration-dependent protection in this model. Of interest, paraquat and $\mathrm{MPP}^{+}$are both parkinsonian toxins and share common toxicity mechanisms, including inhibition of mitochondrial complex 1 activity and dysregulation of autophagy (Dagda et al., 2013), and both compound effects are suppressed by a QR2 inhibitor.

In Vivo Activity: Ultimate Specificity. An ultimate proof of specificity is when enzyme activity is linked to a clear phenotypic outcome (e.g., effects on memory or certain types of memory) in vivo. Here, we found that, on an object-recognition memory test, wild-type mice were sensitive to S29434. After treatment, the animals spent an enhanced period of time exploring the unfamiliar object compared with untreated mice, suggesting a role of QR2 in memory, in keeping with earlier findings (Harada et al., 2001; Benoit et al., 2011; Rappaport et al., 2015). Finally, in the same experiments in QR2 knockout mice, the compound showed no effect. Since the first suggestion of a memory effect (Brouillette and Quirion, 2008), other findings have also linked QR2 catalytic activity to suppression of several types of memory, potentially associated with dopaminergic neurons [McNab et al., 2009; Rossato et al., 2009; Nobili et al., 2017; see also review by D'Amelio et al. (2018)].

Although we did not address S29434 activity in various models of inflammation, which is often linked to ROS production, the inhibitor does not influence Toll-like receptors or inflammasome signaling (Groß et al., 2016) as an activator or inhibitor (O. Gross, personal communication). Further work is needed to better understand a possible role of QR2 in inflammation responses.

In summary, S29434 is a powerful inhibitor of QR2 with an $\mathrm{IC}_{50}$ in the low nanomolar range (5-16, depending on the cosubstrate used). It is specific to QR2 over a range of standard pharmacological targets, is fairly stable in vivo, and penetrates cells. The current findings do not guarantee specificity of S29434 but clearly delineate some of its actions, particularly in various models that help link its activities to its powerful inhibition of QR2. Several other inhibitors of QR2 have been described, such as resveratrol, melatonin, and some flavonoids, but they are nonspecific, inhibiting many enzymes in many pathways; thus, using them to dissect a given pathway is untenable. As such, we believe that the present work lays a strong foundation for the pharmacological use of S29434 to evaluate specifically the role of QR2.

\section{Acknowledgments}

J.A.B. thanks Dr. Hemal Soni (O2h, Ahmedabad, India) for help in writing the second chemical part; San Francisco Edit for their help in editing and rewriting this manuscript; Laure Allain for daily help; and finally, Dr. Philippe Delagrange (Servier, Croissy-sur-Seine, France), Dr. Brian Shilton (London, Ontario, Canada), Dr. Nathaniel Gould, Dr. Kobi Rosenblum (Haifa, Israel), and Olaf Gross (München, Germany) for their intellectual contributions. E.J. thanks Dr. Concetta Martino (Magna Graecia University Catanzaro, Italy) for technical assistance. E.C.H. and P.P.M. thank Cécile Bureau and Laetitia Da Costa for their technical help. Dr. D.A.K. and Dr. K.B. thank Mackenzie Bell, Laura Davidson, and Helen Elizabeth Wallace for their technical assistance.

\section{Authorship Contributions}

Participated in research design: Boutin, Bouillaud, Janda, Gacsalyi, Guillaumet, Hirsch, Kane, Nepveu, Reybier, Bertrand, Le Diguarher, Viaud-Massuard, Ferry.

Conducted experiments: Janda, Reybier, Dupuis, Chhour, Antoine, Brebner, Da Costa, Giganti, Goswani, Guerdouari, Michel, Patel, Paysant, Stojko.

Performed data analysis: Boutin, Bouillaud, Janda, Gacsalyi, Hirsch, Kane, Nepveu, Reybier, Dupuis, Bertrand, Le Diguarher, Antoine, Ducrot, Giganti, Paysant, Stojko, Viaud-Massuard.

Wrote or contributed to the writing of the manuscript: Boutin, Bouillaud, Janda. Gacsalyi, Guillaumet, Hirsch, Kane, Nepveu, Reybier, Dupuis, Le Diguarher, Antoine, Ducrot, Paysant, Stojko, Ferry. 


\section{References}

Alnabulsi S, Hussein B, Santina E, Alsalahat I, Kadirvel M, Magwaza RN, Bryce RA, Schwalbe CH, Baldwin AG, Russo I, et al. (2018) Evaluation of analogues of furanamidines as inhibitors of NQO2. Bioorg Med Chem Lett 28:1292-1297.

Antoine M, Marcheteau E, Delagrange P, Ferry G, and Boutin JA (2012) Characterization of cofactors, substrates and inhibitor binding to flavoenzyme quinone reductase 2 by automated supramolecular nano-electrospray ionization mass spectrometry. Int J Mass Spectrom 312:87-96.

Bartolini L, Casamenti F, and Pepeu G (1996) Aniracetam restores object recognition impaired by age, scopolamine, and nucleus basalis lesions. Pharmacol Biochem Behav 53:277-283.

Benoit CE, Bastianetto S, Brouillette J, Tse Y, Boutin JA, Delagrange P, Wong T, Sarret P, and Quirion R (2010) Loss of quinone reductase 2 function selectively facilitates learning behaviors. J Neurosci 30:12690-12700.

Benoit CE, Rowe WB, Menard C, Sarret P, and Quirion R (2011) Genomic and proteomic strategies to identify novel targets potentially involved in learning and memory. Trends Pharmacol Sci 32:43-52.

Berridge MV, Herst PM, and Tan AS (2005) Tetrazolium dyes as tools in cell biology: new insights into their cellular reduction. Biotechnol Annu Rev 11:127-152.

Bevins RA and Besheer J (2006) Object recognition in rats and mice: a one-trial nonmatching-to-sample learning task to study 'recognition memory'. Nat Protoc 1 1306-1311.

Bhattacharya G, Su T-L, Chia C-M, and Chen K-T (2001) Synthesis and autoxidation of new tetracyclic 9H,10H-indolizino[1,2-b]indole-1-ones. J Org Chem 66:426-432.

Bian J, Li X, Xu L, Wang N, Qian X, You Q, and Zhang X (2017) Affinity-based small fluorescent probe for $\mathrm{NAD}(\mathrm{P}) \mathrm{H}$ :quinone oxidoreductase 1 (NQO1). Design, synthesis and pharmacological evaluation. Eur J Med Chem 127:828-839.

Bindoli A, Rigobello MP, and Galzigna L (1990) Reduction of adrenochrome by rat liver and brain DT-diaphorase. Free Radic Res Commun 8:295-298.

Bolton JL, Trush MA, Penning TM, Dryhurst G, and Monks TJ (2000) Role of quinones in toxicology. Chem Res Toxicol 13:135-160.

Booher J and Sensenbrenner M (1972) Growth and cultivation of dissociated neurons and glial cells from embryonic chick, rat and human brain in flask cultures. $\mathrm{Neu}$ robiology 2:97-105.

Boussard MF, Truche S, Rousseau-Rojas A, Briss S, Descamps S, Droual M, Wierzbicki M, Ferry G, Audinot V, Delagrange P, et al. (2006) New ligands at the melatonin binding site MT(3). Eur J Med Chem 41:306-320.

Boutin JA (2016) Quinone reductase 2 as a promising target of melatonin therapeutic actions. Expert Opin Ther Targets 20:303-317.

Boutin JA, Chatelain-Egger F, Vella F, Delagrange P, and Ferry G (2005) Quinone reductase 2 substrate specificity and inhibition pharmacology. Chem Biol Interact 151:213-228.

Boutin JA and Ferry G (2018) Is there sufficient evidence that the melatonin binding site MT3 is quinone reductase 2? J Pharmacol Exp Ther 368:59-65.

Brouillette J and Quirion R (2008) Transthyretin: a key gene involved in the maintenance of memory capacities during aging. Neurobiol Aging 29:1721-1732.

Buryanovskyy L, Fu Y, Boyd M, Ma Y, Hsieh TC, Wu JM, and Zhang Z (2004) Crystal structure of quinone reductase 2 in complex with resveratrol. Biochemistry 43 : $11417-11426$.

Cassagnes LE, Perio P, Ferry G, Moulharat N, Antoine M, Gayon R, Boutin JA, Nepveu F, and Reybier K (2015) In cellulo monitoring of quinone reductase activity and reactive oxygen species production during the redox cycling of 1,2 and 1,4 quinones. Free Radic Biol Med 89:126-134.

Cassagnes LE, Rakotoarivelo N, Sirigu S, Pério P, Najahi E, Chavas LM, Thompson A, Gayon R, Ferry G, Boutin JA, et al. (2017) Role of quinone reductase 2 in the antimalarial properties of indolone-type derivatives. Molecules 22:E210.

Cecchelli R, Dehouck B, Descamps L, Fenart L, Buée-Scherrer V, Duhem C, Lundquist S, Rentfel M, Torpier G, and Dehouck MP (1999) In vitro model for evaluating drug transport across the blood-brain barrier. Adv Drug Deliv Rev 36:165-178.

Chen D, Li X, Liu X, Liu X, Jiang X, Du J, Wang Q, Liang Y, and Ma W (2017) NQO2 inhibition relieves reactive oxygen species effects on mouse oocyte meiotic maturation and embryo development. Biol Reprod 97:598-611.

Conover TE and Ernster L (1960) Mitochondrial oxidation of extra-mitochondrial TPNH1 mediated by purified DT diaphorase. Biochem Biophys Res Commun 2 26-30.

Dagda RK, Das Banerjee T, and Janda E (2013) How Parkinsonian toxins dysregulate the autophagy machinery. Int J Mol Sci 14:22163-22189.

D'Amelio M, Puglisi-Allegra S, and Mercuri N (2018) The role of dopaminergic midbrain in Alzheimer's disease: translating basic science into clinical practice. Pharmacol Res 130:414-419.

den Braver-Sewradj SP, den Braver MW, Toorneman RM, van Leeuwen S, Zhang Y, Dekker SJ, Vermeulen NPE, Commandeur JNM, and Vos JC (2018) Reduction and scavenging of chemically reactive drug metabolites by $\mathrm{NAD}(\mathrm{P}) \mathrm{H}$ :quinone oxidoreductase 1 and NRH:quinone oxidoreductase 2 and variability in hepatic concentrations. Chem Res Toxicol 31:116-126.

Dufour M, Yan C, Siegel D, Colucci MA, Jenner M, Oldham NJ, Gomez J, Reigan P, Li Y, De Matteis CI, et al. (2011) Mechanism-based inhibition of quinone reductase 2 (NQO2): selectivity for NQO2 over NQO1 and structural basis for flavoprotein inhibition. ChemBioChem 12:1203-1208.

Duncan JS, Gyenis L, Lenehan J, Bretner M, Graves LM, Haystead TA, and Litchfield DW (2008) An unbiased evaluation of CK2 inhibitors by chemoproteomics: characterization of inhibitor effects on CK2 and identification of novel inhibitor targets. Mol Cell Proteomics 7:1077-1088.

Duncan MJ, Takahashi JS, and Dubocovich ML (1988) 2-[125I]iodomelatonin binding sites in hamster brain membranes: pharmacological characteristics and regional distribution. Endocrinology 122:1825-1833.

Ennaceur A and Delacour J (1988) A new one-trial test for neurobiological studies of memory in rats. 1: behavioral data. Behav Brain Res 31:47-59.
Ernster L, Danielson L, and Ljunggren M (1962) DT diaphorase. I. Purification from the soluble fraction of rat-liver cytoplasm, and properties. Biochim Biophys Acta 58:171-188.

Ferry G, Hecht S, Berger S, Moulharat N, Coge F, Guillaumet G, Leclerc V, Yous S, Delagrange $P$, and Boutin JA (2010) Old and new inhibitors of quinone reductase 2 Chem Biol Interact 186:103-109.

Gerard CJJ, Ferry G, Vuillard LM, Boutin JA, Ferte N, Grossier R, Candoni N, and Veesler S (2018) A chemical library to screen protein and protein-ligand crystallization using a versatile microfluidic platform. Cryst Growth Des 18: $5130-5137$.

Groß CJ, Mishra R, Schneider KS, Médard G, Wettmarshausen J, Dittlein DC, Shi H, Gorka O, Koenig P-A, Fromm S, et al. (2016) $\mathrm{K}^{+}$efflux-independent NLRP3 inflammasome activation by small molecules targeting mitochondria. Immunity 45:761-773.

Guerreiro S, Toulorge D, Hirsch E, Marien M, Sokoloff P, and Michel PP (2008) Paraxanthine, the primary metabolite of caffeine, provides protection against dopaminergic cell death via stimulation of ryanodine receptor channels. Mol Pharmacol 74:980-989.

Harada S, Fujii C, Hayashi A, and Ohkoshi N (2001) An association between idiopathic Parkinson's disease and polymorphisms of phase II detoxification enzymes: glutathione S-transferase M1 and quinone oxidoreductase 1 and 2. Biochem Biophys Res Commun 288:887-892.

Janda E, Isidoro C, Carresi C, and Mollace V (2012) Defective autophagy in Parkinson's disease: role of oxidative stress. Mol Neurobiol 46:639-661.

Janda E, Lascala A, Carresi C, Parafati M, Aprigliano S, Russo V, Savoia C, Ziviani E, Musolino V, Morani F, et al. (2015) Parkinsonian toxin-induced oxidative stress inhibits basal autophagy in astrocytes via NQO2/quinone oxidoreductase 2: implications for neuroprotection. Autophagy 11:1063-1080.

Janda E, Parafati M, Aprigliano S, Carresi C, Visalli V, Sacco I, Ventrice D, Mega T, Vadalá N, Rinaldi S, et al. (2013) The antidote effect of quinone oxidoreductase 2 inhibitor against paraquat-induced toxicity in vitro and in vivo. $\mathrm{Br} J$ Pharmacol 168:46-59.

Jiang Y, Liu J, Chen D, Yan L, and Zheng W (2017) Sirtuin inhibition: strategies, inhibitors, and therapeutic potential. Trends Pharmacol Sci 38:459-472.

Kappus H and Sies H (1981) Toxic drug effects associated with oxygen metabolism: redox cycling and lipid peroxidation. Experientia 37:1233-1241.

Knox RJ, Burke PJ, Chen S, and Kerr DJ (2003) CB 1954: from the Walker tumor to NQO2 and VDEPT. Curr Pharm Des 9:2091-2104.

Kuribayashi F, Tsuruta S, Yamazaki T, Nunoi H, Imajoh-Ohmi S, Kanegasaki S, and Nakamura M (2008) Cell adhesion markedly increases lucigenin-enhanced chemiluminescence of the phagocyte NADPH oxidase. Genes Cells 13:1249-1256.

Kuznetsov AV, Veksler V, Gellerich FN, Saks V, Margreiter R, and Kunz WS (2008) Analysis of mitochondrial function in situ in permeabilized muscle fibers, tissues and cells. Nat Protoc 3:965-976.

Kwiek JJ, Haystead TA, and Rudolph J (2004) Kinetic mechanism of quinone oxidoreductase 2 and its inhibition by the antimalarial quinolines. Biochemistry 43: $4538-4547$.

Lascala A, Martino C, Parafati M, Salerno R, Oliverio M, Pellegrino D, Mollace V, and Janda E (2018) Analysis of proautophagic activities of Citrus flavonoids in liver cells reveals the superiority of a natural polyphenol mixture over pure flavones. J Nutr Biochem 58:119-130.

Lavrado J, Paulo A, Gut J, Rosenthal PJ, and Moreira R (2008) Cryptolepine analogues containing basic aminoalkyl side-chains at C-11: synthesis, antiplasmodial activity, and cytotoxicity. Bioorg Med Chem Lett 18:1378-1381.

Leung KK and Shilton BH (2013) Chloroquine binding reveals flavin redox switch function of quinone reductase 2. J Biol Chem 288:11242-11251.

Leung KK and Shilton BH (2015) Quinone reductase 2 is an adventitious target of protein kinase CK2 inhibitors TBBz (TBI) and DMAT. Biochemistry 54:47-59.

Liao S, Dulaney JT, and Williams-Ashman HG (1962) Purification and properties of a flavoprotein catalyzing the oxidation of reduced ribosyl nicotinamide. J Biol Chem 237:2981-2987.

Liao S and Williams-Ashman HG (1961) Enzymatic oxidation of some nonphosphorylated derivatives of dihydronicotinamide. Biochem Biophys Res Commun 4:208-213.

Mailliet F, Ferry G, Vella F, Berger S, Cogé F, Chomarat P, Mallet C, Guénin SP, Guillaumet G, Viaud-Massuard MC, et al. (2005) Characterization of the melatoninergic MT3 binding site on the NRH:quinone oxidoreductase 2 enzyme. Biochem Pharmacol 71:74-88.

Mailliet F, Ferry G, Vella F, Thiam K, Delagrange P, and Boutin JA (2004) Organs from mice deleted for $\mathrm{NRH}$ :quinone oxidoreductase 2 are deprived of the melatonin binding site MT3. FEBS Lett 578:116-120.

McNab F, Varrone A, Farde L, Jucaite A, Bystritsky P, Forssberg H, and Klingberg T (2009) Changes in cortical dopamine D1 receptor binding associated with cognitive training. Science 323:800-802.

Michaelis S, Marais A, Schrey AK, Graebner OY, Schaudt C, Sefkow M, Kroll F, Dreger M, Glinski M, Koester H, et al. (2012) Dabigatran and dabigatran ethyl ester: potent inhibitors of ribosyldihydronicotinamide dehydrogenase (NQO2). J Med Chem 55:3934-3944.

Millan MJ, Mannoury la Cour C, Chanrion B, Dupuis DS, Di Cara B, Audinot V, Cussac D, Newman-Tancredi A, Kamal M, Boutin JA, et al. (2012) S32212, a novel serotonin type $2 \mathrm{C}$ receptor inverse agonist/ $\alpha 2$-adrenoceptor antagonist and potential antidepressant: I. A mechanistic characterization. J Pharmacol Exp Ther 340:750-764.

Nishiyama T, Izawa T, Usami M, Ohnuma T, Ogura K, and Hiratsuka A (2010) Cooperation of NAD(P)H:quinone oxidoreductase 1 and UDP-glucuronosyltransferases reduces menadione cytotoxicity in HEK293 cells. Biochem Biophys Res Commun 394: 459-463.

Nobili A, Latagliata EC, Viscomi MT, Cavallucci V, Cutuli D, Giacovazzo G, Krashia P, Rizzo FR, Marino R, Federici M, et al. (2017) Dopamine neuronal loss 
contributes to memory and reward dysfunction in a model of Alzheimer's disease. Nat Commun 8:14727.

Nosjean O, Ferro M, Coge F, Beauverger P, Henlin JM, Lefoulon F, Fauchere JL, Delagrange P, Canet E, and Boutin JA (2000) Identification of the melatoninbinding site MT3 as the quinone reductase 2. J Biol Chem 275:31311-31317.

Nosjean O, Nicolas JP, Klupsch F, Delagrange P, Canet E, and Boutin JA (2001) Comparative pharmacological studies of melatonin receptors: MT1, MT2 and MT3/ QR2. Tissue distribution of MT3/QR2. Biochem Pharmacol 61:1369-1379.

Nutter LM, Ngo EO, Fisher GR, and Gutierrez PL (1992) DNA strand scission and free radical production in menadione-treated cells. Correlation with cytotoxicity and role of NADPH quinone acceptor oxidoreductase. J Biol Chem 267:2474-2479.

Onyeibor O, Croft SL, Dodson HI, Feiz-Haddad M, Kendrick H, Millington NJ, Parapini S, Phillips RM, Seville S, Shnyder SD, et al. (2005) Synthesis of some cryptolepine analogues, assessment of their antimalarial and cytotoxic activities, and consideration of their antimalarial mode of action. J Med Chem 48:2701-2709.

Paul P, Lahaye C, Delagrange P, Nicolas JP, Canet E, and Boutin JA (1999) Characterization of 2-[125I]iodomelatonin binding sites in Syrian hamster peripheral organs. J Pharmacol Exp Ther 290:334-340.

Pegan SD, Sturdy M, Ferry G, Delagrange P, Boutin JA, and Mesecar AD (2011) Xray structural studies of quinone reductase 2 nanomolar range inhibitors. Protein Sci 20:1182-1195.

Perry CG, Kane DA, Lanza IR, and Neufer PD (2013) Methods for assessing mitochondrial function in diabetes. Diabetes 62:1041-1053.

Perry CG, Kane DA, Lin CT, Kozy R, Cathey BL, Lark DS, Kane CL, Brophy PM, Gavin TP, Anderson EJ, et al. (2011) Inhibiting myosin-ATPase reveals a dynamic range of mitochondrial respiratory control in skeletal muscle. Biochem $J \mathbf{4 3 7}$ : $215-222$.

Pesta D and Gnaiger E (2012) High-resolution respirometry: OXPHOS protocols for human cells and permeabilized fibers from small biopsies of human muscle. Methods Mol Biol 810:25-58.

Polster BM, Nicholls DG, Ge SX, and Roelofs BA (2014) Use of potentiometric fluorophores in the measurement of mitochondrial reactive oxygen species. Methods Enzymol 547:225-250.

Rappaport AN, Jacob E, Sharma V, Inberg S, Elkobi A, Ounallah-Saad H, PasmanikChor M, Edry E, and Rosenblum K (2015) Expression of quinone reductase-2 in the cortex is a muscarinic acetylcholine receptor-dependent memory consolidation constraint. J Neurosci 35:15568-15581.

Reddy PVN, Jensen KC, Mesecar AD, Fanwick PE, and Cushman M (2012) Design, synthesis, and biological evaluation of potent quinoline and pyrroloquinoline ammosamide analogues as inhibitors of quinone reductase 2. J Med Chem 55: 367-377.

Reybier K, Perio P, Ferry G, Bouajila J, Delagrange P, Boutin JA, and Nepveu F (2011) Insights into the redox cycle of human quinone reductase 2. Free Radic Res 45:1184-1195.

Rix U, Hantschel O, Dürnberger G, Remsing Rix LL, Planyavsky M, Fernbach NV, Kaupe I, Bennett KL, Valent P, Colinge J, et al. (2007) Chemical proteomic profiles of the BCR-ABL inhibitors imatinib, nilotinib, and dasatinib reveal novel kinase and nonkinase targets. Blood 110:4055-4063.
Rossato JI, Bevilaqua LRM, Izquierdo I, Medina JH, and Cammarota M (2009) Dopamine controls persistence of long-term memory storage. Science 325:1017-1020. Salthun-Lassalle B, Hirsch EC, Wolfart J, Ruberg M, and Michel PP (2004) Rescue of mesencephalic dopaminergic neurons in culture by low-level stimulation of voltagegated sodium channels. J Neurosci 24:5922-5930.

Sasaki S, Yamada S, Iwamura M, and Kobayashi Y (2013) Specific detection of intramitochondrial superoxide produced by either cell activation or apoptosis by employing a newly developed cell-permeative lucigenin derivative, 10,10' dimethyl-9,9'-biacridinium bis(monomethyl terephthalate). Free Radic Biol Med 65:1005-1011.

Scott KA, Barnes J, Whitehead RC, Stratford IJ, and Nolan KA (2011) Inhibitors of NQO1: identification of compounds more potent than dicoumarol without associated off-target effects. Biochem Pharmacol 81:355-363.

Sorce S, Stocker R, Seredenina T, Holmdahl R, Aguzzi A, Chio A, Depaulis A, Heitz F, Olofsson P, Olsson T, et al. (2017) NADPH oxidases as drug targets and biomarkers in neurodegenerative diseases: what is the evidence? Free Radic Biol Med 112:387-396.

Teixeira G, Szyndralewiez C, Molango S, Carnesecchi S, Heitz F, Wiesel P, and Wood JM (2017) Therapeutic potential of NADPH oxidase $1 / 4$ inhibitors. Br J Pharmacol 174:1647-1669.

Toulorge D, Guerreiro S, Hild A, Maskos U, Hirsch EC, and Michel PP (2011) Neuroprotection of midbrain dopamine neurons by nicotine is gated by cytoplasmic $\mathrm{Ca} 2+$. FASEB J 25:2563-2573.

Troadec J-D, Marien M, Darios F, Hartmann A, Ruberg M, Colpaert F, and Michel PP (2001) Noradrenaline provides long-term protection to dopaminergic neurons by reducing oxidative stress. $J$ Neurochem 79:200-210.

Whittell LR, Batty KT, Wong RPM, Bolitho EM, Fox SA, Davis TME, and Murray PE (2011) Synthesis and antimalarial evaluation of novel isocryptolepine derivatives. Bioorg Med Chem 19:7519-7525.

Wosilait WD and Nason A (1954) Pyridine nucleotide-quinone reductase. I. Purification and properties of the enzyme from pea seeds. J Biol Chem 206:255-270.

Wu K, Knox R, Sun XZ, Joseph P, Jaiswal AK, Zhang D, Deng PS, and Chen S (1997) Catalytic properties of NAD(P)H:quinone oxidoreductase-2 (NQO2), a dihydronicotinamide riboside dependent oxidoreductase. Arch Biochem Biophys 347: 221-228.

Yan C, Dufour M, Siegel D, Reigan P, Gomez J, Shieh B, Moody CJ, and Ross D (2011) Indolequinone inhibitors of NRH:quinone oxidoreductase 2. Characterization of the mechanism of inhibition in both cell-free and cellular systems. Biochemistry 50:6678-6688.

Zhao Q, Yang XL, Holtzclaw WD, and Talalay P (1997) Unexpected genetic and structural relationships of a long-forgotten flavoenzyme to $\mathrm{NAD}(\mathrm{P}) \mathrm{H}$ :quinone reductase (DT-diaphorase). Proc Natl Acad Sci USA 94:1669-1674.

Address correspondence to: Jean A. Boutin, Institut de Recherches Internationales Servier, 50 rue Carnot, 92284 Suresnes, France. E-mail: jean.boutin@servier.com 